\title{
Pain control through selective chemo-axotomy of centrally projecting TRPV1+ sensory neurons
}

\author{
Matthew R. Sapio, ${ }^{1}$ John K. Neubert, ${ }^{2}$ Danielle M. LaPaglia, ${ }^{1}$ Dragan Maric, ${ }^{3}$ Jason M. Keller, ${ }^{1}$ Stephen J. Raithel, ${ }^{1}$ \\ Eric L. Rohrs, ${ }^{2}$ Ethan M. Anderson, ${ }^{4}$ John A. Butman, ${ }^{5}$ Robert M. Caudle, ${ }^{4}$ Dorothy C. Brown, ${ }^{6}$ John D. Heiss, ${ }^{7}$ \\ Andrew J. Mannes, ${ }^{1}$ and Michael J. Iadarola ${ }^{1}$ \\ 'Clinical Center, Department of Perioperative Medicine, NIH, Bethesda, Maryland, USA. ²Department of Orthodontics, University of Florida College of Dentistry, Gainesville, Florida, USA. ${ }^{2}$ Flow Cytometry \\ Core Facility, NIH, National Institute of Neurological Disorders and Stroke, Bethesda, Maryland, USA. ${ }^{4}$ Department of Oral and Maxillofacial Surgery, University of Florida College of Dentistry, Gainesville, \\ Florida, USA. ${ }^{5}$ Clinical Center, Radiology and Imaging Services, NIH, Bethesda, Maryland, USA. ${ }^{6}$ Veterinary Clinical Investigations Center, University of Pennsylvania, School of Veterinary Medicine, \\ Philadelphia, Pennsylvania, USA. 'Turgical Neurology Branch, NIH, National Institute of Neurological Disorders and Stroke, Bethesda, Maryland, USA.
}

\begin{abstract}
Agonists of the vanilloid receptor transient vanilloid potential 1 (TRPV1) are emerging as highly efficacious nonopioid analgesics in preclinical studies. These drugs selectively lesion TRPV1+ primary sensory afferents, which are responsible for the transmission of many noxious stimulus modalities. Resiniferatoxin (RTX) is a very potent and selective TRPV1 agonist and is a promising candidate for treating many types of pain. Recent work establishing intrathecal application of RTX for the treatment of pain resulting from advanced cancer has demonstrated profound analgesia in client-owned dogs with osteosarcoma. The present study uses transcriptomics and histochemistry to examine the molecular mechanism of RTX action in rats, in clinical canine subjects, and in 1 human subject with advanced cancer treated for pain using intrathecal RTX. In all 3 species, we observe a strong analgesic action, yet this was accompanied by limited transcriptional alterations at the level of the dorsal root ganglion. Functional and neuroanatomical studies demonstrated that intrathecal RTX largely spares susceptible neuronal perikarya, which remain active peripherally but unable to transmit signals to the spinal cord. The results demonstrate that central chemo-axotomy of the TRPV1+ afferents underlies RTX analgesia and refine the neurobiology underlying effective clinical use of TRPV1 agonists for pain control.
\end{abstract}

\section{Introduction}

Severe chronic pain remains a medically intractable problem with life-altering consequences. While most chronic pain is currently treated with any number of drugs, including opioids, NSAIDs, and calcium channel modulators, around $40 \%$ of hospital patients report moderate and severe pain (1). Drugs in the opioid class can control moderate to severe pain but also produce side effects such as sedation, respiratory depression, and nausea, and can be highly addictive (2). Pharmacologic silencing of the primary sensory afferents responsible for initial transduction of nociceptive signals with local anesthetics accomplishes profound analgesia for nearly all pain problems but is generally limited by simultaneous actions on nerve fibers subserving other somatosensory modalities and proprioception (3). Additionally, longer-term anesthesia using nerve block approaches often requires chronic infusion with a perineural catheter (3). Systemic pharmacologic analgesics usual-

\section{Authorship note: MRS and JKN contributed equally to this work.}

Conflict of interest: MJI is an inventor on US patent US 8338457 B2, "Selective ablation of pain-sensing neurons by administration of a vanilloid receptor agonist," issued to the $\mathrm{NIH}$, and is on the scientific advisory board of Ark Animal Health. JDH has received research support through a cooperative research and development agreement with Sorrento Therapeutics. JKN, ELR, and RMC are employees of Velocity Laboratories, a company that provides fee-for-service behavioral testing using operant pain assays.

Submitted: April 4, 2017; Accepted: February 1, 2018

Reference information: J Clin Invest. 2018;128(4):1657-1670.

https://doi.org/10.1172/JCI94331. ly act by persistent stimulation of inhibitory receptors or blockade of excitatory ion channels; however, the analgesic actions are frequently accompanied by additional on-target actions due to receptor expression in other regions of the nervous system. Numerous new approaches to systemic methods of pain control are being investigated. Examples include nerve growth factor antibodies (4), biased agonist compounds acting at opioid receptors $(5,6)$, and selective ion channel blockers (7). Despite these advances, pain remains a major concern in clinical care and in the growing opioid crisis (8). In this paper, we examine the mechanisms of resiniferatoxin (RTX) analgesia using multiple methodologies in 3 species with particular attention to the mechanism by which this analgesia is established after different routes of administration.

Localized cell deletion approaches are a permanent and powerful alternative to pharmacologic inhibition of neurons. Regional lesions of peripheral nerve, dorsal root, or trigeminal ganglia with radiofrequency, nonselective chemicals, or neurosurgical interventions are currently in use as treatments for regionalized pain disorders (9-11). While these procedures can be effective, they also have a risk of causing permanent loss of multiple sensations within the innervated region and sensorimotor ataxia due to the loss of proprioceptive inputs (12). With some procedures the pain problem can reoccur despite initial success of the neurosurgical intervention (13). More refined approaches to selectively target pain neurons are in development, including the use of the ultrapotent transient vanilloid potential 1 (TRPV1) agonist RTX to inactivate nocirespon- 
sive primary afferent neurons for veterinary (14-16) and human (17) pain conditions. Because TRPV1 is expressed only in specific subpopulations of primary nociceptive afferents in all species in which this has been carefully examined, this approach is thought to spare most normal nonpainful sensations as well as some pain modalities such as pinch or pinprick, which can be transmitted even after deletion of the TRPV1+ afferents in rodents (14). The extent of the RTX lesion can be regionally delimited by manipulation of the route of administration. Intrathecal application $(18,19)$ can encompass multiple dermatomes, whereas an intraganglionic (14) or periganglionic (20) injection can restrict the lesion to single unilateral segments. Peripheral application under the skin $(21,22)$, adjacent to the nerve $(23,24)$, or intra-articularly $(25,26)$ confines the inactivation to the local nerve terminals, achieving long-term but reversible regional analgesia. These procedure-based applications can be targeted to a patient's pain problem on an individualized basis, and allow for focused pain alleviation with minimal impact on surrounding sensory nerve fibers (18).

The molecular mechanism of RTX-induced pain control is the sustained activation of the nonselective cation channel TRPV1, leading to calcium toxicity of the stimulated compartment of the neuron $(27,28)$. The prolonged elevation of calcium can lead to cell death or compartmentalized structural damage due to calcium toxicity $(28,29)$. Which part of the cell is damaged, and the extent to which cell death is observed, are thought to depend on the concentration and route of administration of RTX. The present study examines these parameters in detail, and informs the clinical use of this approach for pain control. Compared with other vanilloids such as capsaicin, RTX binds more slowly but is a pseudo-irreversible TRPV1 agonist, allowing more calcium to flow through the channel $(28,30)$. In the present work, we show that axotomy of the central portion of the primary afferent axon is a potent mechanism for analgesia following intrathecal RTX administration, and that this central axotomy occurs with preservation of the neuronal perikarya and their peripheral axons, both of which remain functional. Evidence for this comes from examination of differential gene expression in dorsal root ganglia (DRGs) collected from dog and human after treatment with RTX for pain control in cancer, as well as experimental manipulations of the trigeminal ganglion in the rat. Upon administration of RTX into the spinal cerebrospinal fluid (CSF) in a clinical dog trial, and in 1 human patient, we detected only modest changes in DRG gene expression, suggesting that no gross reorganization or large-scale neuronal deletion occurs. In contrast, in vitro bath application of RTX to trigeminal neurons results in significant reduction of several markers of TRPV1 ${ }^{+}$neurons (31). Concurrently, after intrathecal RTX, we observe selective near-total loss of staining for several DRG markers in the corresponding segmental superficial laminae of the dorsal spinal cord, but not in the DRG itself. Taken together, these data demonstrate that loss of the centrally projecting portion of the axon is sufficient to explain pain control when RTX is injected intrathecally. In the rat model, we observed that afferent denervation of the spinal cord is spatially delimited, being near total at the injection site, whereas dorsal root fibers more distal to the injection are preserved. At the same time, the intact peripheral portion of the axon retains the ability to evoke plasma extravasation upon stimulation. Together, these findings demonstrate that intrathecal RTX is mechanistically distinct from intraganglionic or periganglionic applications, which destroy the cell body, leading to destruction of both the central and peripheral branches of the pseudo-unipolar neuron by Wallerian degeneration. Our data support the idea that the selective central axotomy preserves the majority of peripheral responsiveness and transmitter release from the afferent arm of primary nociceptive neurons while effectively blocking transmission of pain signals into the CNS.

\section{Results}

Several data sets were generated and compared to investigate RTX-sensitive DRG neurons. Embryonic TRPV1+ (eTRPV1) neurons were examined by sorting of these cells after RTX-induced activation using FACS. RNA-Seq was performed on eTRPV1 and eIB4-reactive subpopulations $(n=4)$ after sorting, and differential expression analysis was performed between these groups (Supplemental Figures 1-3; supplemental material available online with this article; https://doi.org/10.1172/JCI94331DS1). Adult DRGs were compared with eTRPV1 neurons, which highlighted several critical biological differences between the embryonic and adult DRGs (Supplemental Figure 2). TRPV1 is expressed primarily in sensory ganglia, within 3 subpopulations of neurons previously defined by single-cell RNA-Seq (Supplemental Figure 4 and ref. 32).

Cross-correlation of data sets. Gene expression differences between the treated and untreated groups for each transcriptomic analysis of DRGs exposed to RTX were tabulated. This includes a previously published in vitro ablation experiment (31), which was reanalyzed using MAGIC. In general, there were more genes decreasing than increasing, as expected from a cell deletion approach (Figure 1A). By comparison, in vivo intrathecal RTX administration showed less impact on DRG gene expression (Figure 1, B and C). DRGs from the veterinary dog intrathecal RTX trial showed very few significantly altered genes, indicating that in dog DRGs intrathecal RTX treatment did not cause large-scale cell death and/or affect gene expression. In the human, the lumbar ganglia were compared with cervical ganglia as a within-subject control (Figure 1C). We detected many differentially expressed genes; however, these were most likely due to differences in cervical and lumbar ganglia, as they were not expressed in TRPV1 ${ }^{+}$neurons. In the human patient, injection into the lumbar cistern exposes the lumbar $\mathrm{CSF}$ and dorsal roots in the cauda equina to higher concentrations of drug relative to the cervical rootlets. At 2 weeks after injection this patient had retained thermal sensitivity in his hands to a $41^{\circ} \mathrm{C}$ thermal probe, indicating preservation of TRPV1 ${ }^{+}$fibers at the cervical level (33). While many genes were differentially expressed between these ganglia, this was mainly attributable to higher levels of neurofilament-containing sensory neurons in the cervical ganglia, reflected by the large number of differential genes enriched in these cell types (32). The concordance between these 3 data sets was very poor, with only 1 gene, KLHL1, which encodes the Kelch-like 1 actin-organizing protein, showing a correlated decrease throughout all the data sets (Figure 1D). This analysis includes nonsignificant trends, and as such is conservative and inclusive. Examples of important marker genes are plotted to show the raw data on which these plots are based (Supplemental Figure 5).

To further examine the genes that change in any of the data sets, we examined the profiles of these in several other data sets to 

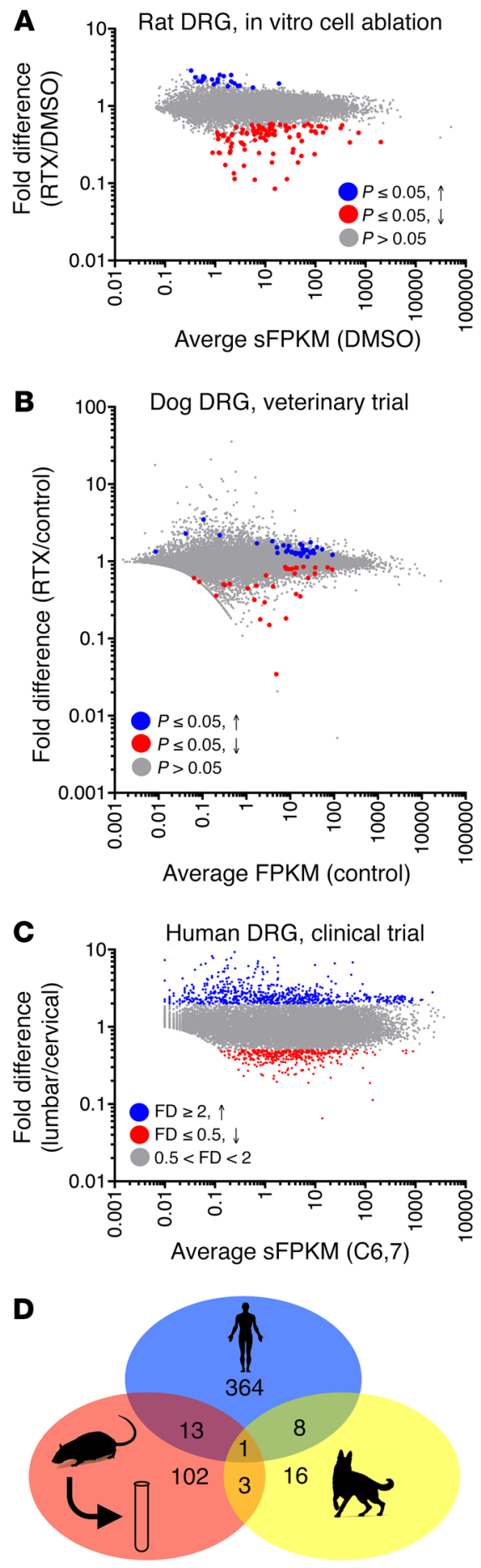

gather more information about their localization. The genes that change tend to be enriched in the DRG relative to the sciatic nerve (Figure 2), suggesting they are enriched in neurons compared with Schwann cells or fibroblasts (34). They also tend to be enriched in the Trpv1-lineage relative to the TRPV1 non-lineage populations (35), and enriched in eTRPV1 small cells relative to eIB4 ${ }^{+}$cells, suggesting that the genes in general are enriched in TRPV1 ${ }^{+}$neurons (Figure 2). The genes changing in the rat and dog experiments also show modest enrichment in TRPV1+ ${ }^{+}$populations of single-cell neurons (Figure 2 and ref. 32).
Figure 1. Summary plots of gene changes after RTX treatment. (A) An in vitro rat study was performed using RTX to ablate TRPV1+ neurons, and RNA-Seq was performed on the remaining cells $(n=3)$. In this experiment cell death was confirmed independently. Significant genes (MAGIC pipeline) for this experiment are colored accordingly. (B) Data from 3 control and 5 RTX-treated companion dogs ( $n=5$ DRGs control, $n=14$ DRGs RTX) enrolled in a veterinary trial that were treated intrathecally with RTX are plotted similarly (statistics in limma, voom). (C) A single human patient was treated with RTX intrathecally in the lumbar region. Data from $\mathrm{C6}, 7$ are plotted against L5. While some differences may exist between these ganglia, there should also be a gradient of RTX action detectable where RTX acts on the TRPV1 ${ }^{+}$neurons in the lumbar DRGs more strongly than in the cervical segments. (D) A Venn diagram of genes changing $40 \%$ or more shows that there is very little overlap between the genes changing in the 3 studies. Only 1 gene, KLHL1 (Kelch-like family member 1 ), encoding an actin-organizing protein, changes by at least $40 \%$ in all 3 data sets.

While few if any genes changed consistently across the data sets, we examined the most strongly correlated changes in detail. We observed that many of the decreasing genes correlated with RTX treatment are in a group designated as the nonpeptidergic 1 subclass. This population most likely has low levels of Trpv1 not detectable in the single-cell data used for these analyses (32), but detectable in a deeper-sequencing approach using fewer cells (ref. 36 and Supplemental Figures 4 and 5). Expression of the Lpar3 gene, which codes for lysophosphatidic acid receptor 3, is decreased in all 3 RTX-treatment data sets. This gene is highly enriched in the nonpeptidergic 1 population in the mouse DRG, and overlaps partially with Trpv1 (Supplemental Figures 6 and 7). A consistent decrease in some genes, including TRPV1, occurs throughout the data sets (Figure 3), although the individual data points are variable (Supplemental Figure 5). The genes encoding TRPV1, KLHL1, and several other proteins are decreased in both the rat in vitro ablation study and the clinical canine and human samples, while genes encoding the protein precursors of calcitonin gene-related peptide (CGRP) and the substance P peptide are reduced in the rat in vitro data sets, but not in the clinical data sets (Supplemental Figure 5), which is consistent with retention of these TRPV1 ${ }^{+}$peptidergic neuronal cell bodies.

Rat staining and plasma extravasation. Dose response for intrathecal injection was explored in the rat (Sprague-Dawley, Harlan Laboratories, or Charles River) using lumbar intrathecal injections and immunohistochemistry in lumbar dorsal spinal cord. Doses around 200 ng of RTX ablated DRG axons in the spinal cord (Supplemental Figure 8). Analysis of sequential rostrocaudal spinal segments in rats injected intra-cisterna magna (ICM) with RTX (250 ng) showed a near-total elimination of TRPV1 staining in the medullary dorsal horn and upper cervical regions consistent with lesioning of the central primary afferent terminals within the nucleus caudalis, but with progressive retention of TRPV1 afferents more caudally. In the same rats, there was a significant decrease in the number of TRPV $1^{+}$neurons following infraorbital nerve (ION), but not ICM, application of RTX, indicating a preservation of cell bodies with ICM but not with ION application (Figure 4, A and B). Similarly, the number of TRPV1 ${ }^{+}$ neurons was not reduced in the DRG after intrathecal injection of 200 ng RTX (Supplemental Figure 8). Delivery of RTX via a bolus injection around the ION or using a Surgicel pellet (Ethicon) 


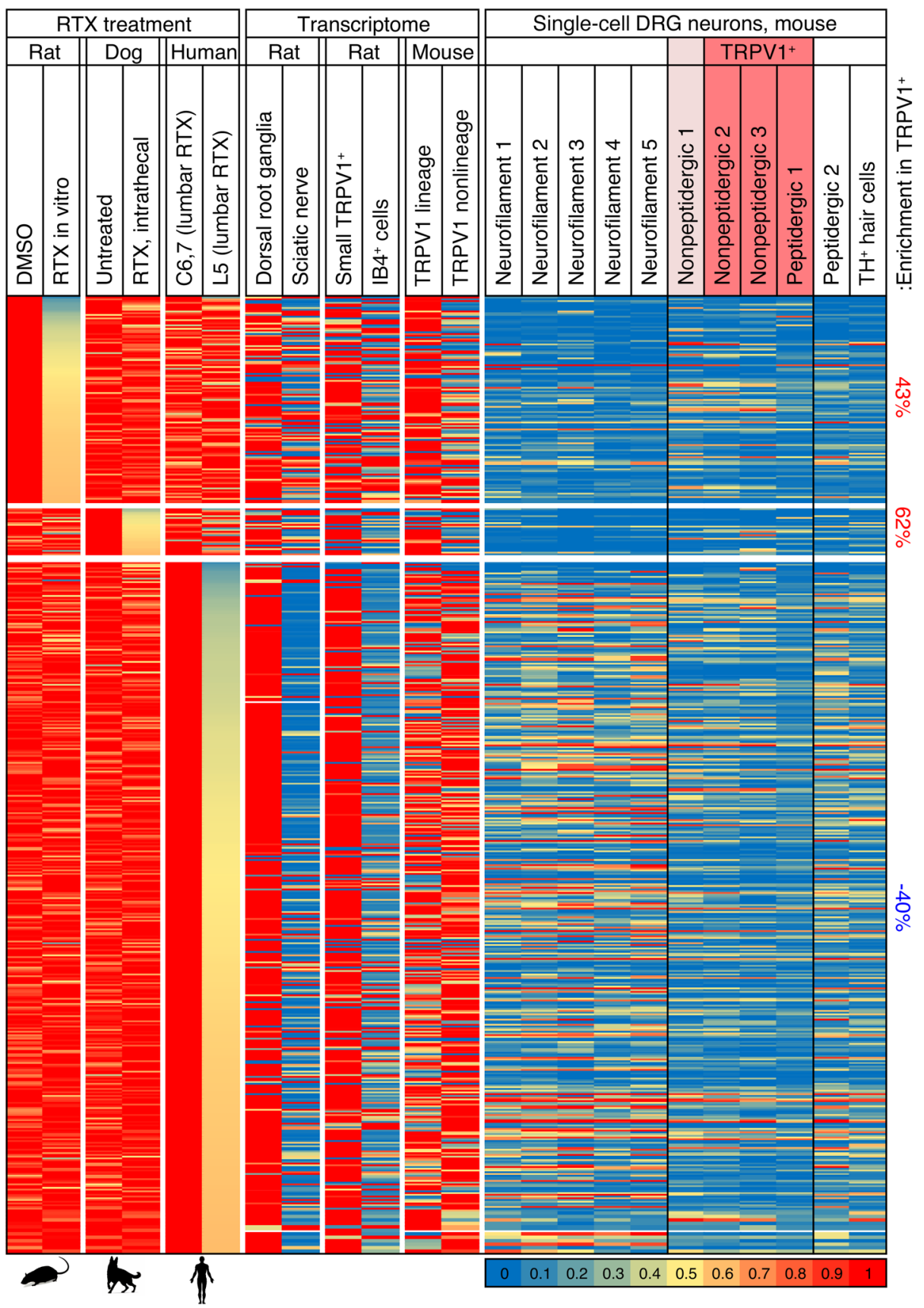

Figure 2. Gene expression map across data sets examining the effects of RTX on gene expression. Data from 7 experiments were mined and analyzed, including 3 experiments in which the TRPV1 agonist RTX was used to selectively damage TRPV1+ neurons. Genes decreasing by at least $40 \%$ are plotted for the 3 experiments in which RTX was used to damage TRPV1 ${ }^{+}$neurons, and compared with several data sets showing differentials between tissues or cell types to indicate where these genes are expressed. In general, these genes are contributed by neurons, as indicated by their enrichment in DRG versus sciatic nerve, and sorted eTRPV1 neurons versus elB4 ${ }^{+}$cells, which includes non-neuronal cells such as microglia and vascular endothelia. Enrichment in TRPV1 ${ }^{+}$neurons in the single-cell data set was tabulated separately. Modest enrichment in these cells was observed in the rat and dog but not human data sets. Values were normalized such that the highest value in any data set is 1 . Data from single cells are normalized differently, according to their original publication. Quantification of enrichment in TRPV1 ${ }^{+}$and nonpeptidergic 1 populations of cells versus other groups is presented. Cenes from rat and dog samples showing a decrease after RTX treatment are enriched in the TRPV $1^{+}$cells. soaked in RTX to isolate the delivery of the drug produced similar significant decreases in cell counts. To test for functional preservation of the cell body and peripheral axon, we examined plasma extravasation using systemic Evans blue dye and topical capsaicin. Capsaicin stimulates neurogenic plasma extravasation, seen as bluing of the skin distinct from the white skin areas that are negative for extravasation (i.e., where TRPV1 ${ }^{+}$fibers are lesioned). We observed that ICM RTX treatment at $250 \mathrm{ng}$ or 2,000 ng did not block plasma extravasation in skin innervated by trigeminal nerves (Figure $4 \mathrm{~F}$ ), indicating that the peripheral nerve terminals were still functional. Using densitometry to analyze images from subregions of the face, we found that 2,000 ng caused a partial blockade of Evans blue extravasation in the area innervated by the maxillary division of the trigeminal (Supple- mental Figure 9). Despite retention of peripheral extravasation at $250 \mathrm{ng}$ RTX, responses to thermal stimulation of the ear were completely eliminated by ICM RTX (Figure 4G). In contrast, ION application of RTX reduced peripheral projections of TRPV1 ${ }^{+}$primary afferents by about $50 \%$ (Figure $4 \mathrm{H}$ ). As a result of the loss of the cells, plasma extravasation was blocked and the skin on the muzzle remained white (Figure $4 \mathrm{~F}$ ). ICM treatment with vehicle did not affect plasma extravasation (Figure 4F).

Magnetic resonance scanning after intrathecal administration of gadolinium contrast agent. In order to more closely examine the spatial relationship between the CSF in the lumbar cistern and the outer sheath of the lumbar ganglia, we examined MRI scans from a patient injected intrathecally with a low concentration of gadolinium contrast agent to track the distribution of the lumbar 


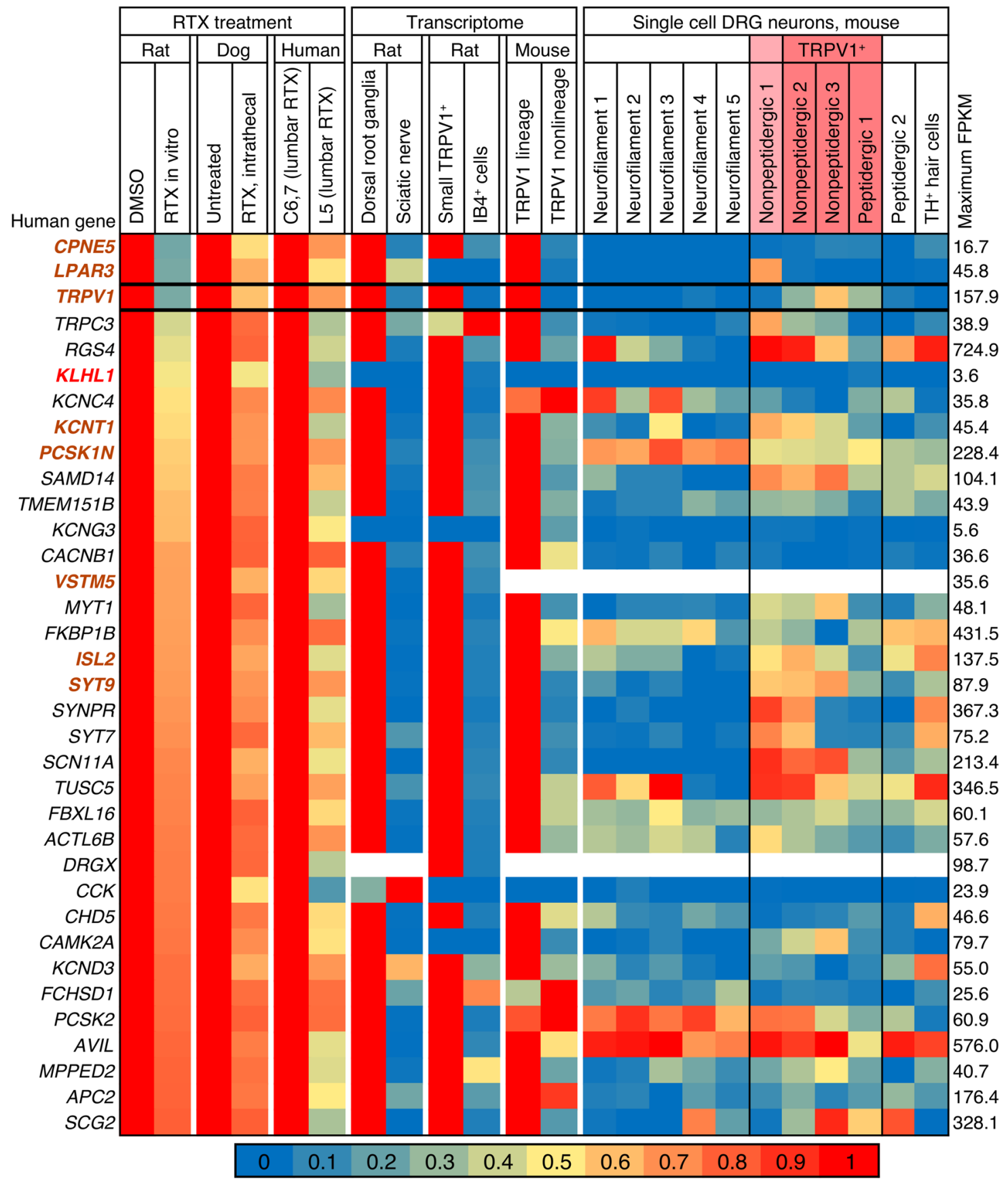

Figure 3. Genes differentially detected in RTX-treated ganglia across experiments in $\mathbf{3}$ species. Data from 7 experiments were mined and analyzed, including 3 experiments in which the TRPV1 agonist RTX was used to selectively damage TRPV1+ neurons in the same manner as in the heatmap in Figure 2. Genes were filtered to show only those genes decreasing by at least $20 \%$ in human, dog, and rat RTX-treated samples. Genes that are also decreased by at least $30 \%$ in all samples are shown in bold orange. The 1 gene (KLHL1) expressed at $\geq 40 \%$ is shown in bold red.

CSF. This scan was chosen because it allowed the nerve fibers in the cauda equina to be distinguished from the ganglia themselves. The bright gadolinium-filled CSF envelops the root sheaths, but extends only to the proximal edge of the DRG, and does not contact the ganglionic mass (Figure 5). In a cross section through the L3 ganglia, no contrast agent is detected penetrating into the DRG capsule (Figure 5, B and C). The presence of contrast in the nerve root sheath but not in the DRG supports the presence of a fluid barrier between the lumbar cistern and the DRG.
Canine and human histology. Two canine studies were performed to evaluate the effects of ICM RTX. Toxicology studies were performed by an independent good laboratory practice (GLP) laboratory as part of an Investigational New Drug application for the usage of RTX in humans. This study was performed in purpose-bred beagles (Harlan Laboratories). A second veterinary trial was conducted in client-owned dogs at the University of Pennsylvania (Supplemental Table 1). In the canine toxicology study, all animals showed normal mental status, gait, and 
A
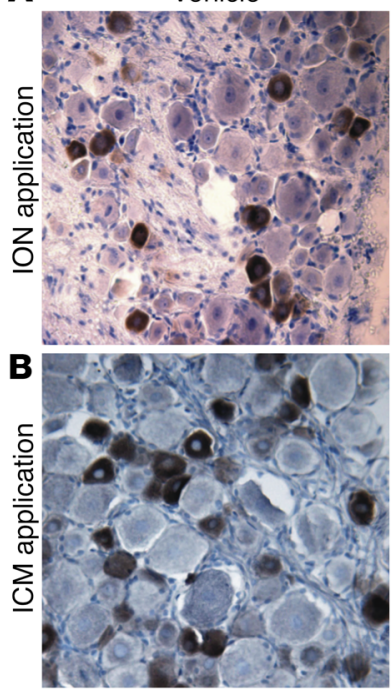

C

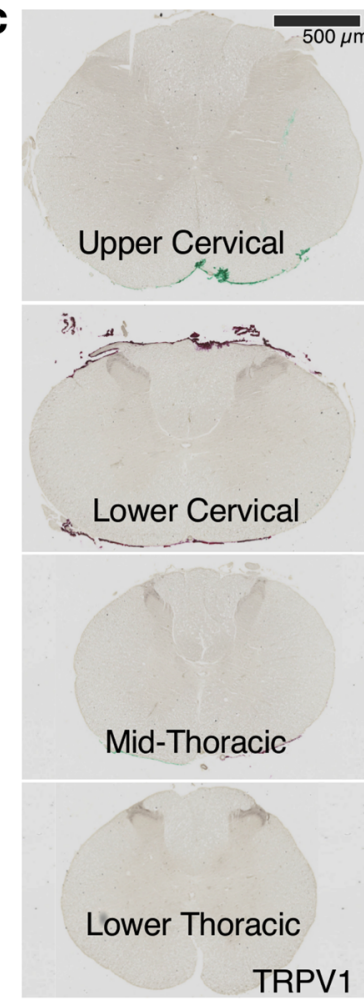

G

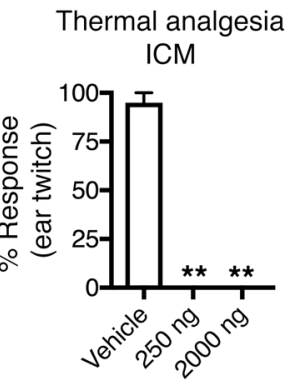

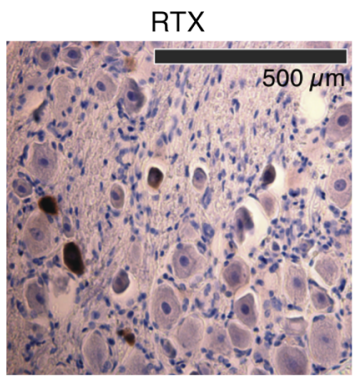
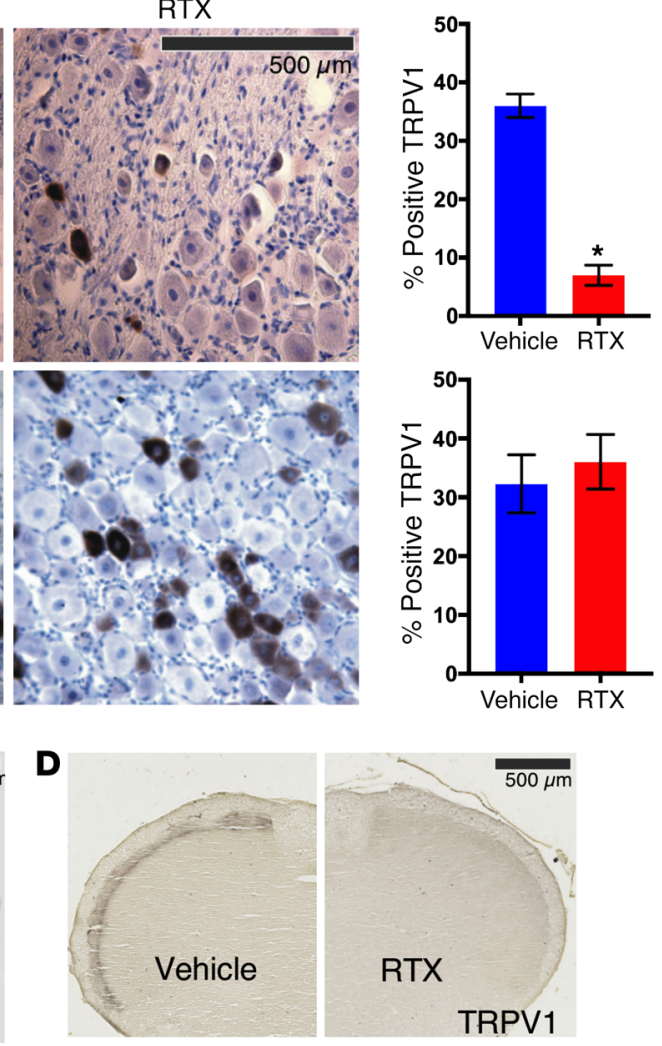

E

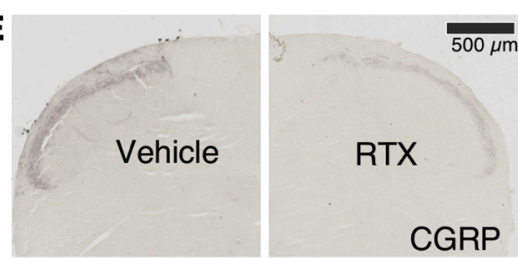

$\mathbf{F}$

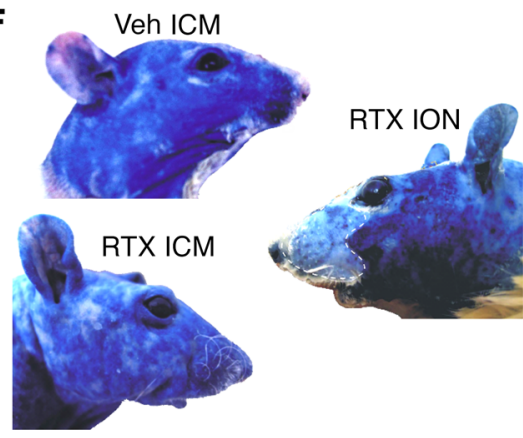

H
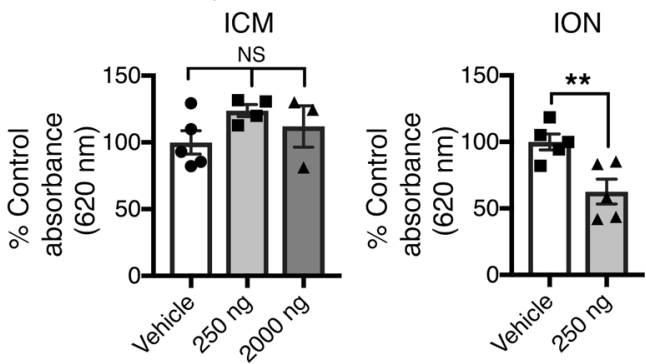

Figure 4. Effects of peripheral versus central targeting of TRPV1 on sensory ganglia. Trigeminal ganglia sections ( $10 \mu \mathrm{m}$, paraffin-embedded) were stained for TRPV1 following $250 \mathrm{ng}$ RTX or vehicle treatment. (A) There was a significant decrease (1-way ANOVA, Scheffé post hoc test, $n=6$ sections, $n=3$ rats, ${ }^{*} P<0.05$ ) in TRPV1 ${ }^{+}$cells in the trigeminal ganglia of rats treated with RTX perineurally around the ION as compared with rats treated with the PBS-vehicle. (B) ICM administration of either RTX or PBS-vehicle did not significantly affect the proportion of cells expressing TRPV1. Counts were completed in various regions of the trigeminal ganglia $\left(V_{1}, V_{2}, V_{3}\right)$, and the proportion of TRPV1+ cells did not vary significantly. (C) ICM injection of RTX reduced TRPV1 staining in the brainstem and upper cervical spinal cord regions by at least $90 \%(n=1)$. Injection of RTX ( $250 \mathrm{ng}$, $10 \mu \mathrm{l})$ produced loss of TRPV $1^{+}$neurons within the upper cervical region, but there was return of staining at the lower cervical/upper thoracic level and regions more caudal. ( $\mathbf{D}$ and $\mathbf{E}$ ) There was a strong reduction in markers of DRG neuronal axons within the brainstem following ICM treatment with RTX $(n=1)$. Additional quantitation is provided in Supplemental Figure 8. (F-H) Peripheral DRG function was tested by examination of capsaicininduced plasma extravasation in trigeminally innervated regions of the face after ICM and ION RTX. While ICM RTX completely blocked thermal responses on the ear (G, ${ }^{* *} P \leq 0.01, n \geq 4$, ANOVA, Dunnett's post hoc), it had no effect on capsaicin-induced extravasation $(\mathbf{H}, n \geq 3$, ANOVA, Dunnett's post hoc). In contrast, ION injection of RTX significantly blocked extravasation $(P \leq 0.05, n=5$, Student's $t$ test).

postural reactions, except one RTX-treated male that was judged "slightly paralytic" on both the pre-dose day and study day 15 , indicating no treatment-related event (Supplemental Table 1). In the client-owned dog study, postinjection increases in heart rate and blood pressure, as reported in prior studies $(15,16)$, were self-limiting and did not require intervention beyond maintenance of general anesthesia until the majority of the autonomic response had subsided. All dogs recovered uneventfully from general anesthesia and were discharged, ambulatory, from the recovery room within 3 hours of extubation. These dogs were discharged from the hospital the following day and reevaluated at 4-week intervals. As in prior studies $(15,16)$, no adverse events associated with RTX injection were noted following hospital discharge.

Dogs treated with ICM RTX were examined histologically. An independent pathologist evaluated trigeminal ganglia and DRGs as part of the toxicology study. At present these reports show no evidence of ganglionic pathology specifically related to RTX treatment $(P=0.1031$ trigeminal; $P=$ 0.0991 DRG; Figure 6, A and B, and Supplemental Table 2). Cervical sections of spinal cords from client-owned dogs were stained for CGRP and substance $P$, and showed a reduction of innervating 

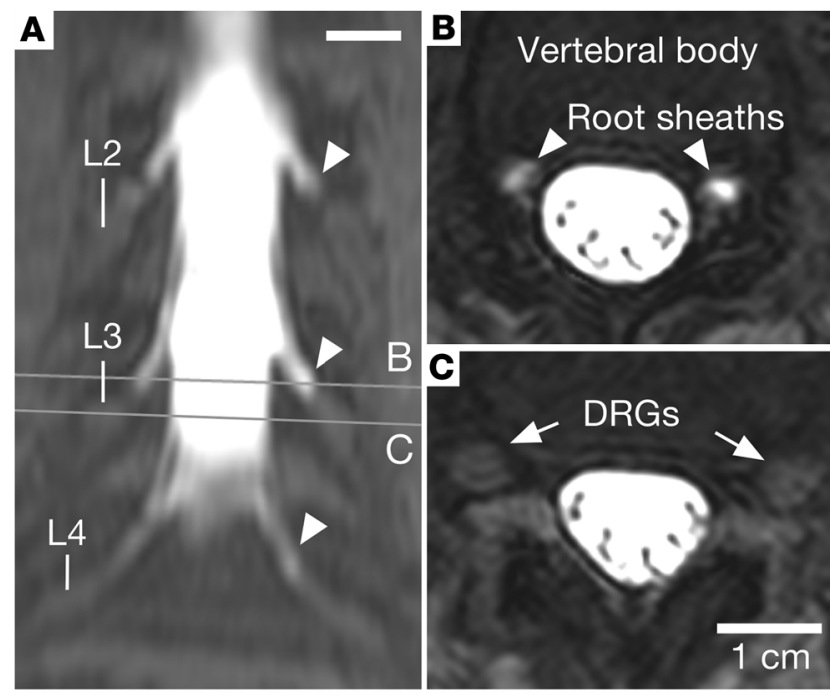

Figure 5. Intrathecal administration of a gadolinium-based contrast agent. An MRI scan was performed as part of a diagnostic evaluation using a dilute amount of gadolinium-based contrast agent injected intrathecally to trace the distribution of CSF in the lumbar region of a single patient. (A) A coronal maximum-intensity projection of T1-weighted fat-saturated MRI shows a high signal in the thecal sac, which extends along nerve root sheaths (arrowheads), without signal within the lumbar ganglia. (B) Axial sections through the lumbar nerve root sheaths (arrowheads) show contrast filling in the nerve root sheaths approaching the DRGs. (C) Axial sections through the DRG (arrows) show no contrast agent in or around the DRGs. Scale bars: $1 \mathrm{~cm}$.

afferents (Figure 6C). Loss of the afferent terminals was observed at the segmental levels corresponding to the point of injection.

The human who received an intrathecal injection in the lumbar region of the spinal cord showed a strong reduction in TRPV1 immunoreactive inputs into the thoracic spinal cord (Figure 6), as well as a reduction in CGRP immunoreactive inputs at both the lumbar and thoracic levels. Substance P immunoreactivity in the lumbar spinal cord was markedly decreased (Figure 6D). The rostral-most section collected from this patient was stained for CGRP alongside a level-matched T6 DRG. Light staining was observed for CGRP in the thoracic spinal cord, with dense staining observable in the DRG neuronal cell bodies (Supplemental Figure 10), indicating that some detectable level of CGRP was present in the thoracic spinal cord, and that CGRP was robustly detectable in the T6 DRG after RTX treatment.

Effects of RTX on reflex-and operant-mediated behaviors in rats. The capsaicin eye wipe response was evaluated as a measure of an unlearned reflexive pain behavior. There was a significant decrease in the number of wipes in rats that were treated with ION RTX (250 ng), and a complete elimination of this response in rats pretreated with ICM RTX (Supplemental Figure 11A). Similar antinociceptive effects were found for mechanical stimuli in hyperalgesic animals (Supplemental Figure 11B).

Operant behavioral assessments provide outcomes that integrate sensory information with cerebral processing (37-40). We used orofacial operant behavioral tasks to assess nociceptive sensitivity of rats pretreated with RTX. In this task, rats were challenged with carrageenan or capsaicin to induce hyperalgesia, which robustly sensitized vehicle-pretreated rats (Supplemental Figure 11, C-E). We observed robust blockade of carrageenan- or capsaicin-induced hyperalgesia by RTX pretreatment (Supplemental Figure 11, C-E).

Dog evaluation and cancer pain analgesia. Three of the six client-owned dogs (50\%) receiving standard-of-care analgesics alone were euthanized before the first 4 -week post-screening evaluation because of increased pain and associated loss of function compared with zero of the 6 RTX-treated dogs. At the time of euthanasia, dogs treated with standard-of-care analgesics alone had significantly higher owner-rated pain scores than dogs that had been treated with RTX (for pain severity score, median 6.8 vs. $3.5,51.5 \%, P=0.01$, Figure 7A; for pain interference score, median 7.5 vs. $5.0,66.7 \%, P=0.006$, Figure $7 \mathrm{~B})$.

Human behavior and analgesic drug use. Pain ratings and opioid consumption were obtained from the human patient over the 7 days before and 15 days after RTX injection. Beginning immediately after RTX treatment, the average Numerical Rating Scale pain rating (1-10 scale) decreased from a mean of 8.3 to a mean of 4.0 (52\% reduction; Figure 7C). Concurrently, the patient spontaneously reduced his opioid use. It was recorded that self-administration of immediate-release oxycodone decreased from 25.7 to $5.3 \mathrm{mg} / \mathrm{d}$ (79.4\% reduction; Figure 7D). Slow-release oxycodone was reduced from an average of 57.1 to $19.3 \mathrm{mg} / \mathrm{d}$ (66.2\% decrease) during the same period (Figure 7E). No significant unanticipated adverse events attributable to RTX treatment were reported. Neurologic examination after RTX injection showed that pinprick and light-touch sensations were normal. Body temperature was not altered by RTX treatment.

\section{Discussion}

TRPV1 agonists have long been known to deplete substance P from primary sensory neurons (41), and to produce prolonged thermal analgesia (42) due to selective degeneration of primary sensory neurons $(43,44)$. However, upon identification of TRPV1 as the endogenous vanilloid receptor (45), the underlying mechanism for the biological action of RTX was identified as calcium cytotoxicity in $\mathrm{TRPV}^{+}$primary afferent nociceptors due to prolonged opening of this $\mathrm{Na}^{+} / \mathrm{Ca}^{++}$cation channel. Unlike capsaicin, RTX binding causes a greatly extended channel opening of TRPV1, leading to rapid cell death $(14,28,46)$. In general, within the nociceptive circuit, cell deletion approaches produce profound analgesia by interrupting the transmission of nociceptive signals either prior to entry into the spinal cord pain circuits or before they ascend out of the spinal cord (14, 47-50). The present paper examines the molecular and neuroanatomical basis for RTX-induced analgesia in rat, dog, and human subjects. Our findings clarify that chemo-axotomy of the centrally projecting axons of TRPV1 $1^{+} \mathrm{DRG}$ neurons is sufficient for profound analgesia. This treatment causes limited sensory neuronal cell death, and few long-term transcriptional changes in sensory neurons. Indeed, the present study, as well as earlier veterinary trials, has shown that administration of RTX into the CSF is effective for pain control in dogs with osteosarcoma $(15,16)$. The efficacy and selectivity of RTX action strongly suggest that these subsets of TRPV1 ${ }^{+} \mathrm{A} \delta$ and $\mathrm{C}$ fiber afferent neurons comprise most of the neurons that transmit clinically relevant pain signals $(14-16,18,19,51)$. 
A
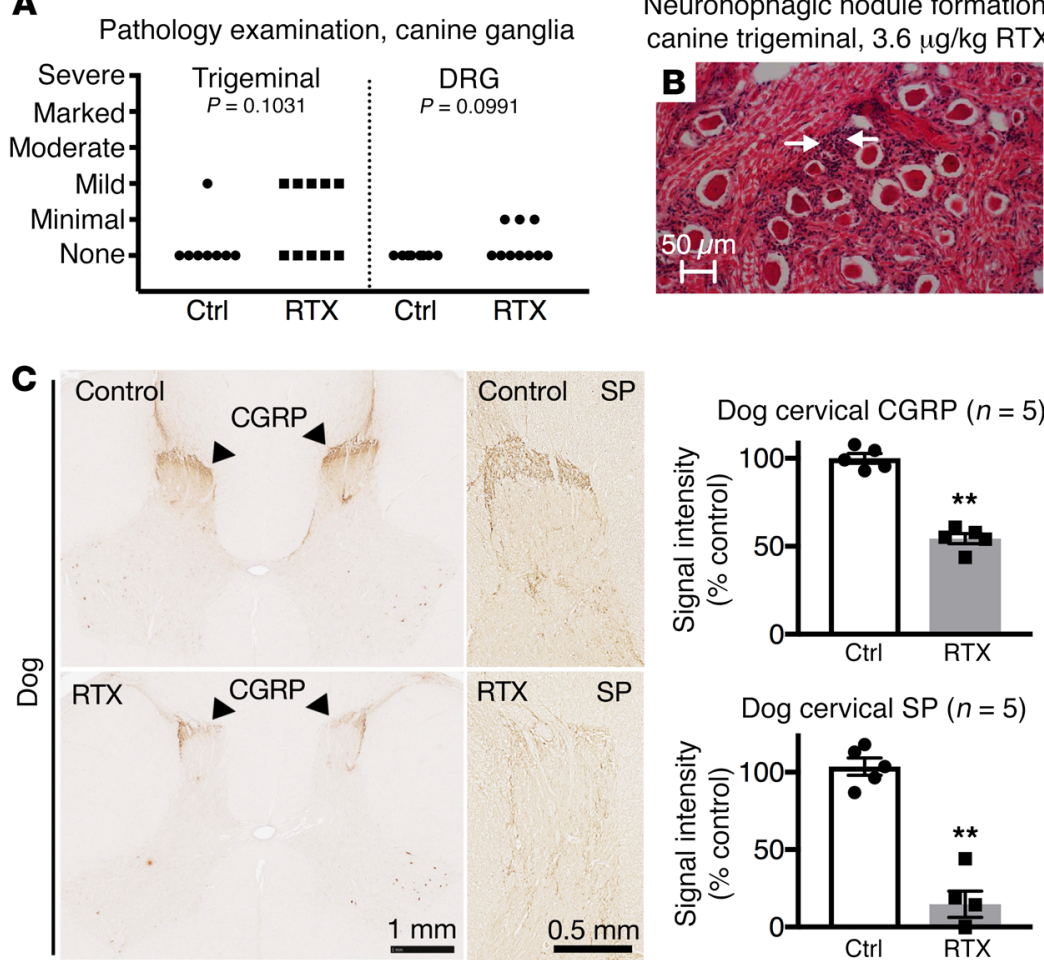
canine trigeminal, $3.6 \mu \mathrm{g} / \mathrm{kg} \mathrm{RTX}$
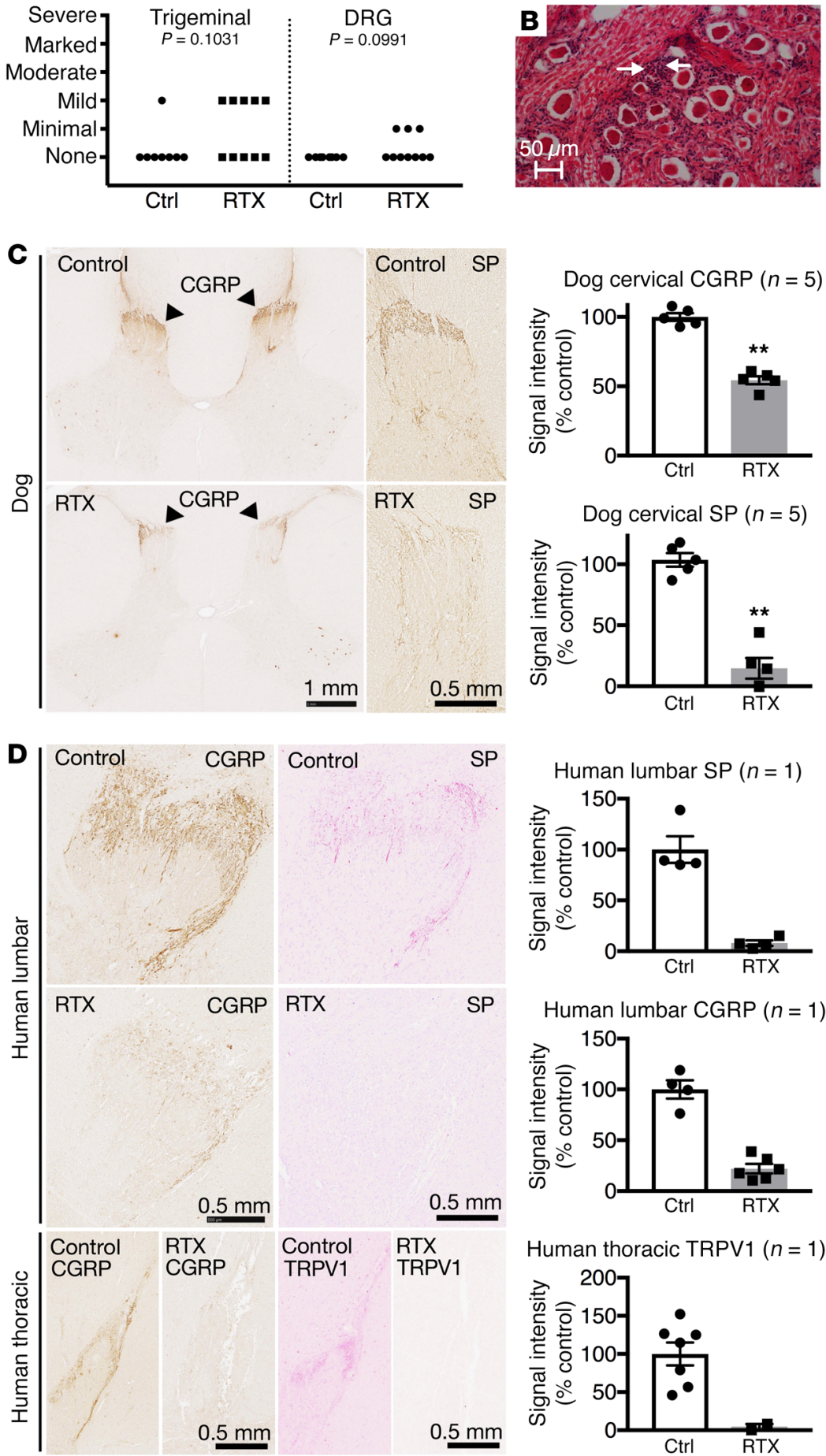

Human thoracic TRPV1 $(n=1)$

Figure 6. Immunohistochemical staining of DRG and spinal cord from human and canine subjects. Dog and human sensory ganglia and spinal cord were harvested after intrathecal treatment with RTX, and immunohistochemical staining was performed. Trigeminal and DRG samples from dogs treated with RTX in an independently conducted GLP toxicology study were assessed by a pathologist. (A) At the maximum tolerated dose $(3.6 \mu \mathrm{g} /$ $\mathrm{kg}), 5$ of 10 dogs treated with RTX showed mild pathology in the trigeminal, whereas 3 of 10 showed minimal pathology in the DRG after ICM injection (nonsignificant, Mantel-Haenszel $\chi^{2}$ ). (B) An example of an infrequent pathological manifestation is the formation of a neuronophagic nodule (arrows). (C) Dogs were injected in the cisterna magna. In the dog, a marked reduction in CGRP was observed in the cervical region of the dorsal spinal cord, with a near-total ablation of substance $P(S P)$ staining at the same level ( $n=5$ dogs). ${ }^{*} P \leq 0.01$. (D) One human patient was injected with RTX using an end-hole catheter positioned at the cauda equina. After RTX treatment, CGRP and SP staining was almost completely abolished in the lumbar spinal cord. Similar reductions were also observed in the thoracic spinal cord for CGRP and TRPV1. A single human was autopsied to procure tissue. To estimate the magnitude of the effect size, several sections of the single human sample (points shown in the graphs) were compared with untreated human sections.

their embryonic developmental stage and express high levels of Ntrk1, the receptor for nerve growth factor, a ligand-receptor system essential for nociceptor survival during development (Supplemental Figure 3). At this developmental stage, the TRPV1+ neurons express only low levels of most adult neuropeptides that participate in the transmission of nociceptive signals, while simultaneously expressing other neuropeptides not present in the adult, further reflecting their immature state (Supplemental Figure 3). The significance of the phenotypic switching between immature and mature DRG neurons is not known at present, but it is consistent with a developmental fine tuning of the DRG neuropeptide repertoire with some peptides being kept in a form of transcriptional reserve status. In this regard, the transcriptomic signature of these neurons will be an informative resource for understanding the transcriptional networks that characterize early life stages of pain cell precursors.

TRPV1 is a nonselective cation channel activated by heat and a number of other exogenous

Because of the importance of TRPV1 neurons in clinical pain, we further explored the lineage and transcriptomic profile of these neurons, by performing RNA-Seq on sorted, functionally activated small-diameter TRPV1 ${ }^{+}$cells from embryonic rats (Supplemental Figure 1). These embryonic TRPV1+ neurons represent a superset of the adult TRPV1+ neurons, as Trpv1 is expressed in the progenitors of several DRG neuronal subtypes, which upon further maturation cease to express Trpv1 (52-54). The E16.5 immature neurons have a transcriptional profile that is characteristic of and endogenous factors (55). In the adult human, TRPV1 is highly enriched in the primary afferent sensory neurons in dorsal root and trigeminal ganglia (Supplemental Figure 4). This anatomical restriction of the molecular drug target is one of the primary factors contributing to RTX specificity. Within mouse DRG primary sensory neurons, Trpv1 is expressed by at least 3 subsets of sensory neurons (32). It is strongly expressed by small-diameter unmyelinated $\mathrm{C}$ fibers as well as some medium-diameter lightly myelinated A $\delta$ fibers $(35,51,56)$. Within these neurons TRPV1 acts 

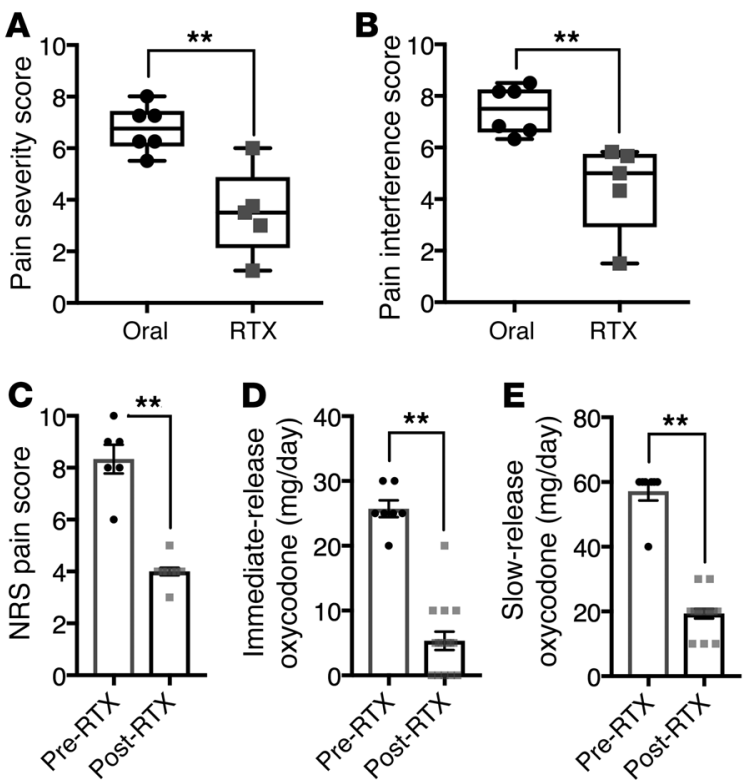

to detect noxious stimuli such as temperature $(45,57)$, by either rapid heating $(\mathrm{A} \delta$ ) or more slow heating (C fiber) (51), and low $\mathrm{pH}$ $(58,59)$, and it is also activated or modulated by endogenous signaling molecules (60-62). Based on these lines of evidence, it is apparent that TRPV1+ afferents are responsive to many forms of noxious stimulation, acting to transduce these stimuli into neural impulses to activate circuits normally involved in escape and protective behaviors. The selective deletion of these fibers by chemical methods eliminates aberrant painful sensations by removing these signals from the circuit.

We report the differentially expressed genes in the dog and human DRG after administration of RTX into the spinal CSF, and compare these findings with other studies that delineate the gene expression profiles of $\mathrm{TRPV}^{+}$neurons $(31,32,35)$. We found a striking lack of overlap between studies in which RTX is used in animals, and studies where TRPV1 ${ }^{+}$DRG neurons are selectively killed. This lack of overlap strongly supports the idea that, at the doses used in the present study, RTX injected into the CSF produces a clinically therapeutic analgesic action while largely sparing the primary afferent perikarya. This is also reflected as modest subsequent gene expression changes in DRGs after intrathecal RTX treatment (Figures 1-3). Sequences from the single human patient were compared between the lumbar and cervical ganglia, which accounts for some noise due to anatomical differences between these ganglia. The dog data are also more variable than the controlled in vitro and in vivo rodent sequencing experiments because of the heterogeneity of the population, breeds, and medical history of the canine clinical population (Figure 1B). However, it is still evident that there is not a substantial, significant decrease in most markers of TRPV1+ DRG neurons. We have previously observed some degree of cell loss, as indicated by the formation of nodules of Nageotte by 30 days in the DRGs of dogs treated with RTX intrathecally (14); however, based on the lack of consistent decreases in DRG transcripts for TRPV1+ neuronal marker genes, we suggest that cell death is not widespread and may occur only in a select, highly sensitive population of densely TRPV1+ neu-
Figure 7. Clinical outcomes in dog and human after RTX treatment. Dogs injected intrathecally with RTX $(n=6)$, and the human patient $(n=1)$ injected intrathecally who was autopsied and whose ganglia were analyzed by RNA-Seq, were assessed for pain control. ( $A$ and $\mathbf{B}$ ) Subsequently to intrathecal RTX treatment, dogs were released to their owners and assessments made at monthly intervals. Owners of each dog rated pain severity and interference with function using the Canine Brief Pain Inventory (78). Pain severity (A) and pain interference (B) scores of companion dogs treated with RTX were significantly decreased relative to those of dogs kept on traditional standard-of-care oral pain medications alone. (C-E) Data from 1 human patient were collected over 7 days before RTX and 15 days after RTX injection while he was an inpatient in the oncology ward. Beginning immediately after RTX treatment, Numerical Rating Scale (NRS) pain scores (1 to 10) decreased from about 8 to about 4 (C). Concurrently, self-administration of immediate-release oxycodone was decreased (D). Slow-release oxycodone was also reduced during the same period (E). Statistical comparisons were made using a 2-tailed Mann-Whitney $U$ test $\left({ }^{*} P<0.01\right.$ ).

rons. These findings are strongly supported by the mild or minimal pathology reports in dogs treated ICM with RTX, and by the sporadic nature of these findings (Figure 6, A and B). We conclude that at this dose tier, there is no evidence of significant sensory neuronal cell loss.

Several genes were decreased in both the rat in vitro study and the clinical dog study of RTX application. These changes could potentially represent loss of specific cells or long-term regulation of transcriptional processes (Figure 3). Of the genes that decrease in all 3 of these data sets, the most strongly differential genes are mainly enriched in TRPV1-lineage neurons and, in most cases, are found in neurons expressing TRPV1 in the adult (Figure 3). One notable exception is Lpar3, which is expressed only by the nonpeptidergic 1 (NP1) subcategory of murine neurons that express Trpv1 at low levels (Figure 3). Detailed analysis reveals that a similar phenomenon applies to Synpr, Trpc3, and Scn11a. However, for each of these genes, colocalization with Trpv1 is greater with deeper sequencing, indicating that detection limits of single-cell sequencing can influence conclusions that can be drawn from this approach (Supplemental Figures 6 and 7). Given the low magnitude and lack of significant changes in these data sets in general (Figures 1 and 2), many of these gene changes may be due to noise. Nonsignificant correlations suggest that NP1 neurons may be affected by RTX treatment despite their relatively low level of TRPV1. We cannot rule out a marginal effect of RTX on these cells. Notably, NP1 neurons selectively express several well-known markers of mechanonociceptive C fibers, such as Mrgprd (32), which does not colocalize well with Trpv1 in costaining experiments (63). Like Lpar3, Mrgprd has some overlap with Trpv1 in single-cell RNA-Seq data, especially in the low-expressing TRPV1 ${ }^{+}$neurons (Supplemental Figure 7). This discrepancy is potentially explained by the more-than-100fold range of expression of Trpv1 in TRPV1+ single DRG neurons (Supplemental Figure 12), making detection of TRPV1 in the lowerexpressing cells difficult. Alternatively, the apparent decreases in genes nominally attributed to the NP1 subpopulation could arise from the fact that these genes are shared between NP1 and the 


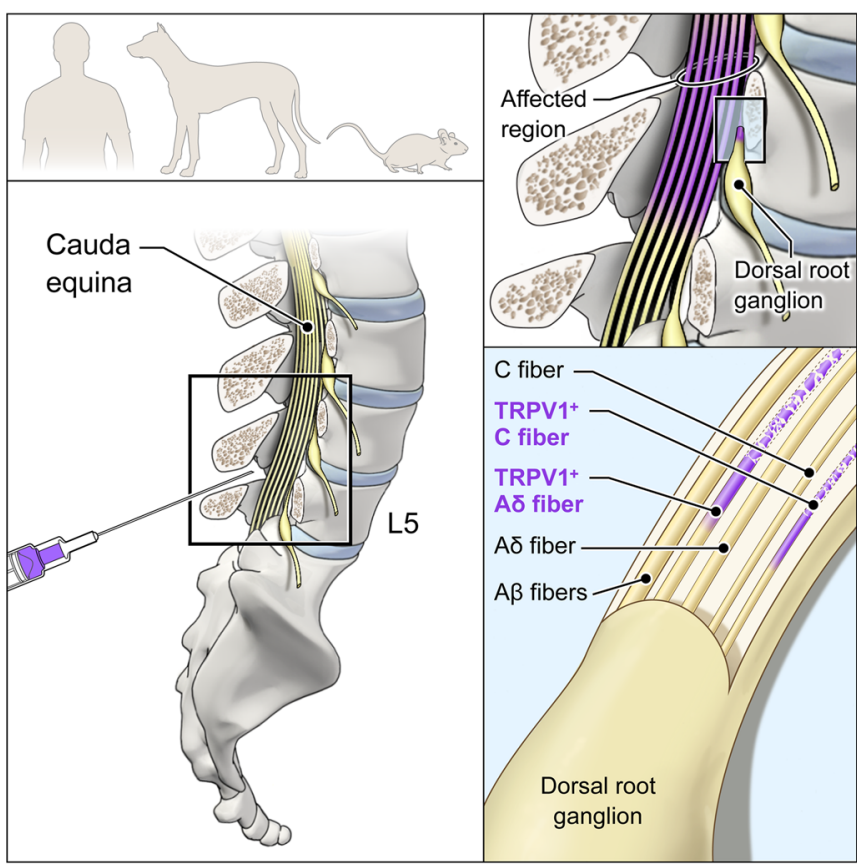

Figure 8. Suggested model of RTX mechanism in rodent, canine, and human. RTX acts at the level of the spinal cord to ablate only the centrally projecting axon of TRPV1-expressing $C$ and $A \delta$ neurons. The neuronal cell bodies in the DRG and the peripherally projecting axonal portion of the neurons are not bathed in drug-containing CSF and remain intact and functional. The TRPV1+ axons are only a subpopulation of the somatosensory and proprioceptive afferent populations. Only the TRPV1 ${ }^{+}$ population is susceptible.

TRPV1+ subclasses of DRG neurons. It is also highly likely that more transcriptionally defined subclasses of neurons will be discovered in the future that more fully elaborate the DRG neuronal repertoire. Despite these caveats to the data set, TRPV1 is among the most strongly reduced genes, presumably because of the loss of the centrally projecting TRPV1+ fibers, which may permanently reduce the total surface area of these neurons. At the neuroanatomical level, there is evidence of cell death occurring in the DRG after intrathecal RTX in companion dogs, based on the formation of nodules of Nageotte 28 days after RTX administration (16). However, the present study shows that these pathological findings are sporadic and difficult to observe consistently, reflecting that intrathecal RTX does not robustly ablate DRG neuronal cell bodies. This is distinct from previous work using intraganglionic or periganglionic RTX, where the mechanism of analgesic action is selective ablation of TRPV1 ${ }^{+}$DRG neurons.

In the rat, ION injections, but not ICM injections, of RTX reduced TRPV1 ${ }^{+}$neurons in the trigeminal ganglia (Figure 4, A and B). The preservation of neuronal cell bodies after ICM injection is consistent with the pathology reports in canine subjects. However, after ICM RTX, staining for TRPV1 ${ }^{+}$nerve terminals decreased at the level of the medullary dorsal horn and upper cervical levels, while the lower cervical and thoracic levels were spared (Figure 6), reflecting a spatially restricted chemo-axotomy of the central axon at the effective dose. At the same time, the neuronal cell bodies and peripherally projecting axons both remained intact and functional, as evidenced by capsaicin-evoked plasma extravasation after ICM but not peripheral administration (ION) of RTX in the rat (Figure $4 \mathrm{~F}$ ). This raises the question of whether intrathecally injected drug can access the DRG cell body. Three-dimensional CT reconstruction in the rat after ICM injection of contrast agent showed enhancement of signal around the cervical spinal cord without enhancement around trigeminal ganglia (64). This same type of limited drug access was evaluated in a single human subject using low-concentration gadolinium dye tracing in the CSF (Figure 5). This unique MRI scan allowed us to examine the relationship between CSF and the DRG, and we were able to visualize that the DRG is not in direct contact with the CSF in this human subject. At the same time, the central axons within the posterior roots were immersed in CSF (Figure 5). While these data were collected from a single patient, the magnetic resonance scan strongly suggests that the DRG is not bathed in CSF, although there may be some individual variation. This is also consistent with intrathecal dye studies using methylene blue in living subjects in which 6 patients were examined, none of whom was observed to have methylene blue in contact with the DRG at autopsy (65). Loss of DRG markers in the rodent dorsal horn after administration of vanilloids intrathecally has been extensively documented $(14,66-68)$. In the dog and human subjects, RTX-induced analgesia is long-lasting if not permanent (refs. 14, 16, 68, and Figure 7). Tissues from these subjects were collected up to 9 months after treatment in companion dogs, and more than 1 month in the human when the patient died of his primary disease. Immunohistochemical evaluation of sections from lumbar spinal cord segments showed loss of neurochemical markers of DRG afferents in the spinal cord, indicative of a persistent lesion of these afferent fibers, and consequently a persistent therapeutic action (Figure 6).

We evaluated both reflex-based nociceptive responses and learned operant behavioral tasks in rats treated with RTX. In all of these tests, ICM RTX was effective at inhibiting behavioral measures of nociception including both capsaicin- and carrageenaninduced hyperalgesia (Supplemental Figure 11). Intrathecal RTX is highly efficacious for pain control, based on data from multiple canine studies (14-16) and further confirmed in the present report (Figure 7). Furthermore, we extend the canine observations to show that RTX-induced ablation of central axons projecting into the superficial dorsal horn leads to a rapid decrease in opioid use and pain scores in 1 human patient treated with intrathecal RTX (Figure 8), from whom we obtained tissue at autopsy for molecular profiling and histology. The combined reduction of pain scores and opioid usage in this patient underscores the efficacy of RTX in controlling cancer pain.

One of the key advantages of RTX is that it can be used with precision injection techniques to target its analgesic actions (19, 20). Additionally, the potency and specificity of the compound allows an effective dose to be confined in a small space with no off-target actions. Intrathecal or ICM injection of RTX can denervate larger regions of the body than more localized injection techniques $(14,20,69)$. However, intrathecal administration also preserves the peripheral axon, allowing for retention of interactions between primary afferent neurons and sites of peripheral innervation, whereas injection methods closer to the soma will kill the cell, thereby eliminating both the peripheral and central endings. Our preclinical data in rats (Supplemental Figure 11 and 
ref. 14), monkeys (69), and client-owned dogs (Figure 7 and refs. $15,16)$ observed for a full year suggest that loss of the TRPV1 neurons produces a sustained analgesic state without producing any observable long-term alteration in sensory processes or sensory dysesthesia leading to aberrant behaviors $(14,16)$. To date, there is no evidence that sensory dysesthesia results from RTX chemoaxotomy. This is potentially a consequence of the anatomical distinctions between this approach and surgical dorsal rhizotomy, which can produce these undesirable effects in humans and in animal models $(70,71)$. The crucial differences between cell deletion and cell axotomy underscore the value of continued investigation into highly specific molecular approaches for pain control.

\section{Methods}

Supplemental Methods are available online with this article; https:// doi.org/10.1172/JCI94331DS1.

Recruitment and demographics of human and canine subjects. Companion dogs were recruited at the University of Pennsylvania as described previously (14-16). Inclusion criteria were appendicular bone cancer confirmed via physical and radiographic examination, as well as a review of history. Exclusion criteria were abnormal serology following complete blood count, clinically significant neurologic disease, or history of unexplained coagulopathy. All dogs were on an analgesic regimen consistent with the standard of care. This included NSAIDs, tramadol, and gabapentin.

The RTX-treated patient was recruited as part of a phase I clinical trial to evaluate the safety of intrathecal RTX for severe refractory pain (ClinicalTrials.gov, NCT00804154). DRGs from this patient were obtained at autopsy 5 weeks after intrathecal RTX injection. Numerical Rating Scale measurements of pain severity (72) and opioid consumption were obtained before and after injection.

Because the transcriptomics indicated a selective effect on the $\mathrm{TRPV}^{+}$dorsal roots, we sought to ascertain the spatial relationships between the lumbar DRGs and intrathecal CSF using a contrastenhanced, high-resolution MRI. This scan was performed on a different patient for diagnostic purposes and used a low concentration (0.5 $\mathrm{ml} ; 0.5 \mathrm{mmol} \mathrm{Gd} / \mathrm{ml} ; 0.25 \mathrm{mmol}$ injected) of the gadolinium contrast agent gadopentetate dimeglumine (Magnevist, Bayer) diluted to $5.0 \mathrm{ml}$ with CSF. Gadolinium contrast was injected intrathecally through the L3-L4 interspace. Scans were obtained on a 1.5 T Philips Achieva MRI using 3D Axial T1 FFE sequence at $0.44 \times 0.44 \times 3.0 \mathrm{~mm}$ resolution.

Transcriptomic analyses. For RNA-Seq studies, frozen tissue was not allowed to thaw and was homogenized immediately after being placed in QIAzol (Qiagen) using the Fast Prep-24 Homogenizer (MP Biologics) according to the manufacturer's protocol. RNA integrity was evaluated by electrophoresis on a 2100 Bioanalyzer with an RNA 6000 Nano Kit (Agilent). Illumina sequencing was performed at Beckman Coulter (canine) or the NIH Intramural Sequencing Center (rat, human) as described previously $(22,34,73)$. Expression values were mined directly from previously published resources (32, $34,35,73,74)$. FASTQ files previously published in ref. 31 were mined and reanalyzed. Rat and human data sets were aligned using MAGIC software (75) with genomic targets based on Rn6 (rat) and GRCh37 (human) with RefSeq annotations. Data sets generated for this article are available through the Sequence Read Archive under BioProjects PRJNA428250, PRJNA428348, and PRJNA432439. For each bulk tissue data set, expression values were calculated as SFPKM (significant fragments per kilobase of transcript per million mapped reads; MAGIC pipeline) (73) or FPKM (Quality of RNA-seq Tool-Set [QoRTs; National Human Genome Research Institute] quantification). Single-cell expression data (32) are expressed as the fraction of positive cells within each DRG neuronal subclass (0 to 1). Analysis of single-cell data was performed using published algorithms (Rtsne package, R).

A ratio was calculated by division of the experimental and control groups by the maximum value in either case. In order to focus on strongly expressed genes, if the maximum value of sFPKM/FPKM across both data sets was $\leq 1$, indicating it was not strongly expressed in any tissue examined, these values were not analyzed. Filtering was performed to identify genes decreasing by $40 \%, 30 \%$, or $20 \%$ in the 3 data sets where RTX was used to ablate or chemo-axotomize TRPV1+ neurons.

Peripheral and central delivery of $R T X$ in rats. We targeted the trigeminal nociceptive circuit via 2 percutaneous approaches: (a) from the peripheral end of the primary afferent, and (b) centrally at the primary afferent/second-order neuron synapse. RTX or vehicle was injected perineurally around the infraorbital nerve (ION), a major branch of the maxillary division $\left(\mathrm{V}_{2}\right)$ of the trigeminal nerve. The most anterorostral portion of the zygomatic process of the maxillary bone was palpated, and then the 25-gauge needle was inserted perpendicular and through the skin medial to this point. The needle was advanced until the bony surface of the maxillary bone was encountered and then was withdrawn about $1 \mathrm{~mm}$ before injecting RTX (50 $\mu \mathrm{l}, 250 \mathrm{ng}$ ) or vehicle (0.25\% Tween-80 in PBS, $0.05 \%$ ascorbic acid).

Central administration of RTX or vehicle was achieved by injection into the cisterna magna under isoflurane anesthesia, as described previously (76). Briefly, animals were maintained at $2 \%-3 \%$ isoflurane, and a 28-gauge 0.5-cc insulin syringe was carefully directed so as to touch the middle to lower portion of the occiput and then gradually moved caudally until the needle punctured the atlanto-occipital membrane overlying the cisterna magna. To check placement, a small amount of CSF was aspirated; then RTX or vehicle $(10 \mu \mathrm{l})$ was injected slowly $(1 \mu \mathrm{l} / \mathrm{s})$. Rats were allowed to recover for at least 1 week before behavioral testing.

Neurogenic inflammation induced plasma extravasation. The extent of plasma extravasation following capsaicin-induced neurogenic inflammation was evaluated in a subset of rats 1 week after ICM or perineural RTX or vehicle injection. Following anesthesia (2:1 ketamine/xylazine, i.p.), the hair from the orofacial region was completely removed using a depilatory cream (Nair, Carter-Wallace Inc.). Evans blue (Sigma-Aldrich) was infused (30 mg/kg; $2 \%$ solution, i.v.) via tail vein or femoral vein injection, capsaicin cream (5\%) was liberally and evenly applied to the face, and digital photographs were captured (Canon PowerShot SD550). For colorimetric measurements, superficial skin samples were collected, and Evans blue dye was extracted in formamide and quantified as previously described (14).

Immunohistochemical staining. Rat trigeminal ganglia samples were paraffin-processed and cut to $10-\mu \mathrm{m}$ sections. Following deparaffinization and epitope unmasking with Target Retrieval Solution (S1700, Dako) at $95^{\circ} \mathrm{C}$ for 20 minutes, sections were blocked with $10 \%$ normal goat serum (S-1000, Vector Laboratories Inc.) and incubated overnight at $4^{\circ} \mathrm{C}$ with the TRPV1 primary antibody (1:10,000; Oncogene, PC420) or a carboxy-amide terminally directed antibody against rat $\alpha$ CGRP (1:15,000; generated by Michael J. Iadarola, NIH, Bethesda, Maryland, USA) (77). Antibody detection was performed using the VECTASTAIN Elite Rabbit IgG and Peroxidase Substrate 
Kits (SK-4700 and SK-4100, Vector Laboratories Inc.) and 3,3'-diaminobenzidine tetrahydrochloride (DAB). Histological sections were visualized with an Olympus BX 60 microscope equipped with an RT Slider CCD camera and processed with Spot Advanced software. A blinded observer chose 2 nonconsecutive trigeminal sections for each group (treated vs. nontreated), and the number of TRPV1 immunoreactive and nonimmunoreactive cell bodies within a rectangular reticule was counted by visual inspection ( $\times 10$ magnification); the ratio of $\mathrm{TRPV}^{+}$neurons to the total number of neurons was calculated.

Frozen dog cervical spinal cord was cut into 5- to 7-mm segments and postfixed in $10 \%$ formalin overnight. Human and dog spinal cords were embedded in paraffin, and 6- $\mu \mathrm{m}$ sections were cut. The slides were warmed at $60^{\circ} \mathrm{C}$ for 20 minutes, deparaffinized in xylenes 3 times for 5 minutes each, and hydrated in a decreasing EtOH gradient. Antigen retrieval was performed in a 1,200-W microwave for 3 minutes at $100 \%$ power and 5 minutes at $30 \%$ power. Slides were incubated in goat blocking serum [VECTASTAIN Elite ABC HRP Kit (Peroxidase, Rabbit IgG), Vector Laboratories Inc.] for 30 minutes. The slides were incubated in primary antibody $(\alpha C G R P, 1: 10,000$, Peninsula Laboratories, T-4239; substance P, 1:1,000, ImmunoStar, 20064; TRPV1, 1:5,000, Aves Labs, Tigard OR, custom) (20) diluted in PBS with 1\% BSA, $0.05 \%$ sodium azide, and $0.1 \%$ Tween-20 for 45 minutes (dog) or 1 hour (human). The slides were incubated in biotinylated secondary antibody from the VECTASTAIN Kit for 30 minutes, incubated in the Vector ABC Reagent for 30 minutes, and developed with the ImmPACT DAB kit (Vector Laboratories Inc.) with hematoxylin counterstain (1:10,000; Sigma-Aldrich). Slides were digitized using a Hamamatsu NanoZoomer 2.0HT Digital Slide Scanner before quantification.

Injection procedures and behavioral testing in companion dogs and human subject. Eleven client-owned dogs with unilateral osteosarcoma were analyzed in this study. Five were treated with RTX 10 days after screening, and 6 dogs with osteosarcoma served as behavioral controls and continued to receive standard-of-care analgesics alone; only 4 of the latter group were available for tissue analysis. For intrathecal RTX injection, under supervision of a board-certified veterinary anesthesiologist, general anesthesia was induced according to standard of care considering dog breed, age, and anesthesiologist preferences (Supplemental Table 1). The dogs were intubated and anesthesia was maintained with isoflurane in oxygen at 1.5 mean alveolar concentration. Dogs were monitored with continuous ECG, pulse oximetry, and indirect arterial blood pressure. Chlorhexidine solution was used for sterile preparation of the intrathecal injection site. A 20-gauge 1.5-inch spinal needle (BD spinal needle with Quincke bevel, Becton Dickinson and Co.) was placed percutaneously into the CSF space at the level of the cisterna magna for dogs with forelimb tumors, and a 22-gauge 3-inch needle was introduced percutaneously to the subarachnoid space between L5 and L6 for dogs with hind-limb tumors. Flow of clear CSF confirmed proper needle placement, and then 1.2 $\mu \mathrm{g} / \mathrm{kg}$ RTX $(100 \mu \mathrm{g} / \mathrm{ml}$ solution) was injected into the intrathecal space, followed by sterile saline $(0.1 \mathrm{ml} / \mathrm{kg}$ for ICM injections or 0.5 $\mathrm{ml}$ for lumbar injections). After injection, the head and neck were elevated approximately 30 degrees above the posterior half of the body to inhibit cranial flow of RTX. At 60 minutes after injection, dogs were maintained on increasingly lower concentrations of isoflurane based on changes in heart rate and blood pressure until they could be safely extubated. The dogs were assessed for adverse events, and the Canine Brief Pain Inventory (BPI) was administered at monthly intervals until the dog's death. The Canine BPI contains 4 questions pertaining to the severity of pain (the averaged responses for these questions result in a pain severity score [PSS]) and 6 questions pertaining to how the pain interferes with the dog's routine activities (the averaged responses for these questions result in a pain interference score [PIS]). As long as the same owner provides all assessments for his or her dog throughout the study, the Canine BPI provides a valid and reliable owner assessment of chronic pain (78).

In our ongoing human clinical trial of RTX for intractable cancer pain, we were able to obtain DRG and spinal cord at autopsy from 1 male patient. The participant was monitored using cardiac electrodes throughout the pre-, peri-, and postoperative periods. Sinus tachycardia was present throughout all phases of the operative procedure, and the patient was treated with a total of $3 \mathrm{mg}$ metoprolol i.v. to decrease heart rate within baseline limits. Supplemental oxygen was administered with nasal cannula at the beginning of the procedure and switched to an oxygen mask on transfer to the postanesthesia care unit. Hemodynamics were monitored at 15 -minute intervals through a noninvasive blood pressure cuff maintained throughout the procedure and postoperatively. This patient was injected with $13 \mu \mathrm{g}$ RTX in $2 \mathrm{ml}$ of vehicle at the L4-L5 interspace under propofol anesthesia with intermittent fentanyl (50-75 $\mu \mathrm{g})$. Dose justification was based on preclinical studies in canine osteosarcoma $(14,16)$, and in consultation with the chair of the National Cancer Institute Protocol Monitoring and Review Committee. Hydralazine (10 mg i.v.) was given once to address the rise in blood pressure at the time of RTX injection. After treatment, the participant was medicated with a total of 4 doses of hydromorphone ranging from 0.2 to $0.4 \mathrm{mg}$ each within approximately 1 hour of arrival in the postanesthesia care unit. Metoprolol ( $2 \mathrm{mg}$ i.v. twice) was once again administered to treat sinus tachycardia after the procedure. The patient died of his primary disease 5 weeks after RTX injection, at which time lumbar, thoracic, and cervical ganglia and spinal cord samples were removed and stored at $-80^{\circ} \mathrm{C}$ until RNA extraction or immersion fixation in $4 \%$ paraformaldehyde for histology.

Canine toxicology study. An independent GLP canine toxicology study was performed at Bridge GPS Inc. as part of the Investigational New Drug application for RTX. The cohort of animals consisted of 8 controls (4 male, 4 female), 8 animals treated at $1.2 \mu \mathrm{g} / \mathrm{kg}$ ( 4 male, 4 female), and 10 treated at $3.6 \mu \mathrm{g} / \mathrm{kg}$ (6 male, 4 female). In this study, purpose-bred beagles (Harlan Laboratories) were injected with 1.2 $\mu \mathrm{g} / \mathrm{kg}$ RTX, or the maximum tolerated dose of $3.6 \mu \mathrm{g} / \mathrm{kg} \mathrm{RTX}$, intracisternally. All animals in the study were assessed using a functional observational battery at predosing, as well as at study days 2 and 15 . Subsequently, trigeminal and dorsal root ganglia tissue was collected from animals treated with $3.6 \mu \mathrm{g} / \mathrm{kg}$ RTX and assessed by a pathologist and assigned scores.

Statistics. For RNA-Seq data sets analyzed using the MAGIC pipeline, differential scores were generated and compared with Wilcoxon signed-rank $P$ values, as previously described (75). Wilcoxon signedrank $P$ values less than 0.05 are considered significant. Dog DRG samples were analyzed using RNA-STAR (https://github.com/alexdobin/ STAR) and the CanFam3.1 genome with Ensembl (https://uswest. ensembl.org/index.html) annotations. Gene expression profiles were generated from 3 control and 5 RTX-treated companion dogs ( $n=5$ DRGs control, $n=14$ DRGs RTX). Quantification and quality control of the dog DRG RNA-Seq data sets were carried out using QoRTs (79), with statistical analyses performed using linear modeling in voom 
(limma). A $P$ value of less than 0.05 was considered significant using this test. For the immunohistochemical TRPV1 comparison (Figure 4, A and B), a 1-way ANOVA and Scheffé post hoc test was used to evaluate treatment effects on the percentage of TRPV1 $1^{+}$cells within the trigeminal ganglia. For the pathologist's evaluation of canine sensory ganglia, a Mantel-Haenszel $\chi^{2}$ test was applied (Figure 6C). Data in Figure 7 were compared using a 2-tailed Mann-Whitney $U$ test ${ }^{*} P<$ 0.01). For human data in Figure 7, statistical comparisons were made using data from a single patient over multiple time points before and after RTX treatment. Data points represent measurements from different time points, and not different individuals. Error bars throughout the figures represent mean \pm SEM.

Study approval. The companion dog protocol and informed consent document were approved by the Institutional Animal Care and Use Committee and the Privately-Owned Animal Protocol committee at the University of Pennsylvania. The canine toxicology study was performed at Bridge GPS Inc. in accordance with their standard operating procedures. In rat studies, all care, handling, and behavioral testing were approved by the Institutional Animal Care and Use Committee at the University of Florida or the NIH, and all procedures complied with the Guide for the Care and Use of Laboratory Animals (National Academies Press, 2011). Human RNA-Seq and MRI data were from studies approved by the Combined Neurosciences Institutional Review Board of the NIH Intramural Research Program. Written informed consent was obtained from the dog owners and the human subjects prior to inclusion in the studies. Dose justification for intrathecal RTX was based on preclinical studies in canine osteosarcoma, and in consultation with the chair of the National Cancer Institute Protocol Monitoring and Review Committee.

\section{Author contributions}

MRS, JKN, AJM, and MJI conceptualized the project, and drafted the manuscript. MRS, JKN, DML, DM, JMK, SJR, ELR, EMA, JAB, RMC, DCB, JDH, AJM, and MJI collected and analyzed data. MRS, JMK, DML, SJR, JAB, DCB, and MJI revised the manuscript.

\section{Acknowledgments}

The authors thank Stephen Hewitt and Kris Ylaya for the use of the Hamamatsu slide scanner, Ethan Smith for his help with bioinformatics, and Ruth Yaskovich for her help with immunohistochemistry. The authors thank Jacklyn Gross for her help with RNA extraction. This work was supported by the intramural research programs of the $\mathrm{NIH}$, Clinical Center, and NIH grants 1K22DE014865-01A1, 5R21DE016704-02, and K08DA017720. This work was also supported by funding from the National Center for Complementary and Integrative Health (1ZIAAT000017-03).

Address correspondence to: Matthew R. Sapio, Department of Perioperative Medicine, Clinical Center, National Institutes of Health, Building 10, Room 2C401, 10 Center Drive, MSC 1510, Bethesda, Maryland 20892-1510, USA. Phone: 301.594.4041; Email:matt.sapio@nih.gov.
1. Carr EC, Meredith P, Chumbley G, Killen R, Prytherch DR, Smith GB. Pain: a quality of care issue during patients' admission to hospital. J Adv Nurs. 2014;70(6):1391-1403.

2. Deyo RA, Von Korff M, Duhrkoop D. Opioids for low back pain. BMJ. 2015;350:g6380.

3. Ilfeld BM. Continuous peripheral nerve blocks: a review of the published evidence. Anesth Analg. 2011;113(4):904-925.

4. Hefti FF, et al. Novel class of pain drugs based on antagonism of NGF. Trends Pharmacol Sci. 2006;27(2):85-91.

5 . Kingwell K. Pioneering biased ligand offers efficacy with reduced on-target toxicity. Nat Rev Drug Discov. 2015;14(12):809-810.

6. White KL, et al. The G protein-biased $\kappa$-opioid receptor agonist $\mathrm{RB}-64$ is analgesic with a unique spectrum of activities in vivo.J Pharmacol Exp Ther. 2015;352(1):98-109.

7. Bregman $\mathrm{H}$, et al. Identification of a potent, state-dependent inhibitor of Nav1.7 with oral efficacy in the formalin model of persistent pain. JMed Chem. 2011;54(13):4427-4445.

8. Federal Pain Research Strategy - Final Report. NIH Website. https://iprcc.nih.gov/. Accessed February 15, 2018.

9. Abejón D, Garcia-del-Valle S, Fuentes ML, Gómez-Arnau JI, Reig E, van Zundert J. Pulsed radiofrequency in lumbar radicular pain: clinical effects in various etiological groups. Pain Pract. 2007;7(1):21-26.

10. Boswell MV, et al. Interventional techniques: evidence-based practice guidelines in the management of chronic spinal pain. Pain Physician. 2007;10(1):7-111.
11. Raslan AM, Cetas JS, McCartney S, Burchiel KJ. Destructive procedures for control of cancer pain: the case for cordotomy. J Neurosurg. 2011;114(1):155-170

12. Krinke G, Heid J, Bittiger H, Hess R. Sensory denervation of the plantar lumbrical muscle spindles in pyridoxine neuropathy. Acta Neuropathol. 1978;43(3):213-216.

13. Lee A, McCartney S, Burbidge C, Raslan AM, Burchiel KJ. Trigeminal neuralgia occurs and recurs in the absence of neurovascular compression. J Neurosurg. 2014;120(5):1048-1054.

14. Karai L, et al. Deletion of vanilloid receptor 1-expressing primary afferent neurons for pain control. J Clin Invest. 2004;113(9):1344-1352.

15. Brown DC, Agnello K, Iadarola MJ. Intrathecal resiniferatoxin in a dog model: efficacy in bone cancer pain. Pain. 2015;156(6):1018-1024.

16. Brown DC, et al. Physiologic and antinociceptive effects of intrathecal resiniferatoxin in a canine bone cancer model. Anesthesiology. 2005;103(5):1052-1059.

17. Heiss JD, et al. Intrathecal resiniferatoxin for intractable cancer pain. J Neurosurg. 2015;123(2):A515-A.

18. Iadarola MJ, Gonnella GL. Resiniferatoxin for pain treatment: an interventional approach to personalized pain medicine. Open Pain J. 2013;6:95-107.

19. Iadarola MJ, Mannes AJ. The vanilloid agonist resiniferatoxin for interventional-based pain control. Curr Top Med Chem. 2011;11(17):2171-2179.

20. Brown JD, et al. CT-guided injection of a TRPV1 agonist around dorsal root ganglia decreases pain transmission in swine. Sci Transl Med. 2015;7(305):305ra145.

21. Salas MM, Clifford JL, Hayden JR, Iadarola MJ, Averitt DL. Local resiniferatoxin induces longlasting analgesia in a rat model of full thickness thermal injury. Pain Med. 2017;18(12):2453-2465.

22. Raithel SJ, Sapio MR, LaPaglia DM, Iadarola MJ, Mannes AJ. Transcriptional changes in dorsal spinal cord persist after surgical incision despite preemptive analgesia with peripheral resiniferatoxin. Anesthesiology. 2018;128(3):620-635.

23. Neubert JK, et al. Perineural resiniferatoxin selectively inhibits inflammatory hyperalgesia. $\mathrm{Mol}$ Pain. 2008;4:3.

24. Kissin I, Davison N, Bradley EL. Perineural resiniferatoxin prevents hyperalgesia in a rat model of postoperative pain. Anesth Analg. 2005;100(3):774-780.

25. Kim Y, et al. The effects of intra-articular resiniferatoxin on monosodium iodoacetate-induced osteoarthritic pain in rats. Korean J Physiol Pharmacol. 2016;20(1):129-136.

26. Kissin EY, Freitas CF, Kissin I. The effects of intraarticular resiniferatoxin in experimental knee-joint arthritis. Anesth Analg. 2005;101(5):1433-1439.

27. Szallasi A, Blumberg PM. Resiniferatoxin, a phorbol-related diterpene, acts as an ultrapotent analog of capsaicin, the irritant constituent in red pepper. Neuroscience. 1989;30(2):515-520.

28. Kárai LJ, Russell JT, Iadarola MJ, Oláh Z. Vanilloid receptor 1 regulates multiple calcium compartments and contributes to $\mathrm{Ca}^{2+}$-induced $\mathrm{Ca}^{2+}$ release in sensory neurons. J Biol Chem. 2004;279(16):16377-16387.

29. Grant ER, Dubin AE, Zhang SP, Zivin RA, Zhong 
Z. Simultaneous intracellular calcium and sodium flux imaging in human vanilloid receptor 1 (VR1)-transfected human embryonic kidney cells: a method to resolve ionic dependence of VR1-mediated cell death. JPharmacol Exp Ther. 2002;300(1):9-17.

30. Olah Z, et al. Ligand-induced dynamic membrane changes and cell deletion conferred by vanilloid receptor 1.J Biol Chem. 2001;276(14):11021-11030.

31. Isensee J, Wenzel C, Buschow R, Weissmann R, Kuss AW, Hucho T. Subgroup-elimination transcriptomics identifies signaling proteins that define subclasses of TRPV1-positive neurons and a novel paracrine circuit. PLoS One. 2014;9(12):e115731.

32. Usoskin D, et al. Unbiased classification of sensory neuron types by large-scale single-cell RNA sequencing. Nat Neurosci. 2015;18(1):145-153.

33. Ran C, Hoon MA, Chen X. The coding of cutaneous temperature in the spinal cord. Nat Neurosci. 2016;19(9):1201-1209.

34. Sapio MR, Goswami SC, Gross JR, Mannes AJ, Iadarola MJ. Transcriptomic analyses of genes and tissues in inherited sensory neuropathies. Exp Neurol. 2016;283(pt A):375-395.

35. Goswami SC, et al. Molecular signatures of mouse TRPV1-lineage neurons revealed by RNA-Seq transcriptome analysis. J Pain. 2014;15(12):1338-1359.

36. Li CL, et al. Somatosensory neuron types identified by high-coverage single-cell RNAsequencing and functional heterogeneity. Cell Res. 2016;26(8):967.

37. Neubert JK, Widmer CG, Malphurs W, Rossi HL, Vierck CJ Jr, Caudle RM. Use of a novel thermal operant behavioral assay for characterization of orofacial pain sensitivity. Pain. 2005;116(3):386-395.

38. Neubert JK, Rossi HL, Malphurs W, Vierck CJ Jr, Caudle RM. Differentiation between capsaicin-induced allodynia and hyperalgesia using a thermal operant assay. Behav Brain Res. 2006;170(2):308-315.

39. Anderson EM, et al. Use of the Operant Orofacial Pain Assessment Device (OPAD) to measure changes in nociceptive behavior.J Vis Exp. 2013;(76):e50336.

40. Murphy NP, Mills RH, Caudle RM, Neubert JK. Operant assays for assessing pain in preclinical rodent models: highlights from an orofacial assay. Curr Top Behav Neurosci. 2014;20:121-145.

41. Jessell TM, Iversen LL, Cuello AC. Capsaicininduced depletion of substance $P$ from primary sensory neurones. Brain Res. 1978;152(1):183-188.

42. Yaksh TL, Farb DH, Leeman SE, Jessell TM. Intrathecal capsaicin depletes substance $P$ in the rat spinal cord and produces prolonged thermal analgesia. Science. 1979;206(4417):481-483.

43. Jancsó G, Kiraly E, Jancsó-Gábor A. Pharmacologically induced selective degeneration of chemosensitive primary sensory neurones. Nature. 1977;270(5639):741-743.

44. Oh U, Hwang SW, Kim D. Capsaicin activates a nonselective cation channel in cultured neonatal rat dorsal root ganglion neurons. J Neurosci. 1996;16(5):1659-1667.

45. Caterina MJ, Schumacher MA, Tominaga M,
Rosen TA, Levine JD, Julius D. The capsaicin receptor: a heat-activated ion channel in the pain pathway. Nature. 1997;389(6653):816-824

46. Olah Z, Karai L, Iadarola MJ. Protein kinase $\mathrm{C}$ (alpha) is required for vanilloid receptor 1 activation. Evidence for multiple signaling pathways. J Biol Chem. 2002;277(38):35752-35759.

47. Alvarez P, Gear RW, Green PG, Levine JD. IB4-saporin attenuates acute and eliminates chronic muscle pain in the rat. Exp Neurol. 2012;233(2):859-865.

48. Mantyh PW, et al. Inhibition of hyperalgesia by ablation of lamina I spinal neurons expressing the substance P receptor. Science. 1997;278(5336):275-279.

49. Vulchanova L, Olson TH, Stone LS, Riedl MS, Elde R, Honda CN. Cytotoxic targeting of isolectin IB4-binding sensory neurons. Neuroscience. 2001;108(1):143-155.

50. Benoliel R, Eliav E, Mannes AJ, Caudle RM, Leeman S, Iadarola MJ. Actions of intrathecal diphtheria toxin-substance $P$ fusion protein on models of persistent pain. Pain. 1999;79(2-3):243-253.

51. Mitchell K, Lebovitz EE, Keller JM, Mannes AJ, Nemenov MI, Iadarola MJ. Nociception and inflammatory hyperalgesia evaluated in rodents using infrared laser stimulation after Trpv1 gene knockout or resiniferatoxin lesion. Pain. 2014;155(4):733-745.

52. Mishra SK, Tisel SM, Orestes P, Bhangoo SK, Hoon MA. TRPV1-lineage neurons are required for thermal sensation. EMBO J. 2011;30(3):582-593.

53. Cavanaugh DJ, Chesler AT, Bráz JM, Shah NM, Julius D, Basbaum AI. Restriction of transient receptor potential vanilloid-1 to the peptidergic subset of primary afferent neurons follows its developmental downregulation in nonpeptidergic neurons. J Neurosci. 2011;31(28):10119-10127.

54. Le Pichon CE, Chesler AT. The functional and anatomical dissection of somatosensory subpopulations using mouse genetics. Front Neuroanat. 2014;8:21.

55. Pingle SC, Matta JA, Ahern GP. Capsaicin receptor: TRPV1 a promiscuous TRP channel. Handb Exp Pharmacol. 2007;(179):155-171.

56. Mitchell K, et al. Ablation of rat TRPV1-expressing Adelta/C-fibers with resiniferatoxin: analysis of withdrawal behaviors, recovery of function and molecular correlates. Mol Pain. 2010;6:94.

57. Gavva NR, et al. The vanilloid receptor TRPV1 is tonically activated in vivo and involved in body temperature regulation. J Neurosci. 2007;27(13):3366-3374

58. Dhaka A, et al. TRPV1 is activated by both acidic and basic pH. J Neurosci. 2009;29(1):153-158.

59. Kaszas K, et al. Small molecule positive allosteric modulation of TRPV1 activation by vanilloids and acidic $\mathrm{pH}$. J Pharmacol Exp Ther. 2012;340(1):152-160.

60. Cao E, Cordero-Morales JF, Liu B, Qin F, Julius D. TRPV1 channels are intrinsically heat sensitive and negatively regulated by phosphoinositide lipids. Neuron. 2013;77(4):667-679.

61. Ross RA. Anandamide and vanilloid TRPV1 receptors. Br JPharmacol. 2003;140(5):790-801.

62. Olah Z, Karai L, Iadarola MJ. Anandamide activates vanilloid receptor 1 (VR1) at acidic $\mathrm{pH}$ in dorsal root ganglia neurons and cells ectopically expressing VR1. J Biol Chem. 2001;276(33):31163-31170.

63. Zylka MJ, Rice FL, Anderson DJ. Topographically distinct epidermal nociceptive circuits revealed by axonal tracers targeted to Mrgprd. Neuron. 2005;45(1):17-25.

64. Rossi HL, Vierck CJ Jr., Caudle RM, Yezierski RP, Neubert JK. Dose-dependent effects of icilin on thermal preference in the hindpaw and face of rats. J Pain. 2009;10(6):646-653

65. Somberg HM. The relation of the spinal subarachnoid and perineurial spaces. JNeuropathol Exp Neurol. 1947;6(2):166-171.

66. Gamse R, Petsche U, Lembeck F, Jancsò G. Capsaicin applied to peripheral nerve inhibits axoplasmic transport of substance $P$ and somatostatin. Brain Res. 1982;239(2):447-462.

67. Cavanaugh DJ, et al. Distinct subsets of unmyelinated primary sensory fibers mediate behavioral responses to noxious thermal and mechanical stimuli. Proc Natl Acad Sci U S A. 2009;106(22):9075-9080.

68. Jeffry JA, Yu SQ, Sikand P, Parihar A, Evans MS, Premkumar LS. Selective targeting of TRPV1 expressing sensory nerve terminals in the spinal cord for long lasting analgesia. PLoS One. 2009;4(9):e7021.

69. Tender GC, et al. Selective ablation of nociceptive neurons for elimination of hyperalgesia and neurogenic inflammation. J Neurosurg. 2005;102(3):522-525

70. Levitt M. Dysesthesias and self-mutilation in humans and subhumans: a review of clinical and experimental studies. Brain Res. 1985;357(3):247-290.

71. Basbaum AI. Effects of central lesions on disorders produced by multiple dorsal rhizotomy in rats. Exp Neurol. 1974;42(3):490-501.

72. Childs JD, Piva SR, Fritz JM. Responsiveness of the numeric pain rating scale in patients with low back pain. Spine. 2005;30(11):1331-1334

73. LaPaglia DM, et al. RNA-Seq investigations of human post-mortem trigeminal ganglia [published online ahead of print July 12, 2017]. Cephalalgia. https://doi.org/10.1177/0333102417720216.

74. GTEx Consortium. The Genotype-Tissue Expression (GTEx) project. Nat Genet. 2013;45(6):580-585.

75. Zhang W, et al. Comparison of RNA-seq and microarray-based models for clinical endpoint prediction. Genome Biol. 2015;16:133.

76. Neubert JK, et al. Characterization of mouse orofacial pain and the effects of lesioning TRPV1expressing neurons on operant behavior. Mol Pain. 2008;4:43.

77. Traub RJ, Iadarola MJ, Ruda MA. Effect of multiple dorsal rhizotomies on calcitonin gene-related peptide-like immunoreactivity in the lumbosacral dorsal spinal cord of the cat: a radioimmunoassay analysis. Peptides. 1989;10(5):979-983.

78. Brown DC, Boston R, Coyne JC, Farrar JT. A novel approach to the use of animals in studies of pain: validation of the canine brief pain inventory in canine bone cancer. Pain Med. 2009;10(1):133-142.

79. Hartley SW, Mullikin JC. QoRTs: a comprehensive toolset for quality control and data processing of RNA-Seq experiments. BMC Bioinformatics. 2015;16:224. 